\title{
Differential gene expression among three sex types reveals a MALE STERILITY 1 (CpMS1) for sex differentiation in papaya
}

\author{
Dessireé Zerpa-Catanho' ${ }^{1}$ Jennifer Wai ${ }^{1}$, Ming Li Wang ${ }^{2}$, Li'ang Yu' ${ }^{1}$, Julie Nguyen ${ }^{1}$ and Ray Ming ${ }^{1 *}$
}

\begin{abstract}
Background: Carica papaya is a trioecious plant species with a genetic sex-determination system defined by sex chromosomes. Under unfavorable environmental conditions male and hermaphrodite exhibit sex-reversal. Previous genomic research revealed few candidate genes for sex differentiation in this species. Nevertheless, more analysis is still needed to identify the mechanism responsible for sex flower organ development in papaya.
\end{abstract}

Results: The aim of this study was to identify differentially expressed genes among male, female and hermaphrodite flowers in papaya during early (pre-meiosis) and later (post-meiosis) stages of flower development. RNA-seq was used to evaluate the expression of differentially expressed genes and RT-qPCR was used to verify the results. Putative functions of these genes were analyzed based on their homology with orthologs in other plant species and their expression patterns. We identified a Male Sterility 1 gene (CpMS1) highly up-regulated in male and hermaphrodite flower buds compared to female flower buds, which expresses in small male flower buds (3-8 mm), and that might be playing an important role in male flower organ development due to its homology to MS1 genes previously identified in other plants. This is the first study in which the sex-biased expression of genes related to tapetum development in the anther developmental pathway is being reported in papaya. Besides important transcription factors related to flower organ development and flowering time regulation, we identified differential expression of genes that are known to participate in ABA, ROS and auxin signaling pathways (ABA-8-hydroxylases, AIL5, UPBEAT 1, VAN3-binding protein).

Conclusions: CPMS1 was expressed in papaya male and hermaphrodite flowers at early stages, suggesting that this gene might participate in male flower organ development processes, nevertheless, this gene cannot be considered a sex-determination gene. Due to its homology with other plant MS1 proteins and its expression pattern, we hypothesize that this gene participates in anther development processes, like tapetum and pollen development, downstream gender specification. Further gene functional characterization studies in papaya are required to confirm this hypothesis. The role of ABA and ROS signaling pathways in papaya flower development needs to be further explored as well.

Keywords: Carica papaya, Flower development, Male sterility gene, RT-qPCR, RNA-Seq, Sex differentiation

\footnotetext{
* Correspondence: rayming@illinois.edu

${ }^{1}$ Department of Plant Biology, University of Illinois at Urbana-Champaign,

Urbana, IL 61801, USA

Full list of author information is available at the end of the article
}

(c) The Author(s). 2019 Open Access This article is distributed under the terms of the Creative Commons Attribution 4.0 International License (http://creativecommons.org/licenses/by/4.0/), which permits unrestricted use, distribution, and reproduction in any medium, provided you give appropriate credit to the original author(s) and the source, provide a link to the Creative Commons license, and indicate if changes were made. The Creative Commons Public Domain Dedication waiver (http://creativecommons.org/publicdomain/zero/1.0/) applies to the data made available in this article, unless otherwise stated. 


\section{Background}

Unisexual flowers in angiosperm plant species are classified as monoecious or dioecious. Monoecious plant species have female and male flowers in separate flowers but on the same individual (6\% angiosperm species), while dioecious species have female and male flowers on separate individuals (5\% angiosperm species). Dioecious plant species could evolve from hermaphroditic or monoecious populations in three major steps $[1,2]$. First, a recessive male-sterile mutation occurred originating female plants. The occurrence of this mutation would be advantageous for the population, because female plants could be pollinated by individuals from different populations, reducing the inbreeding and increasing the genetic variability within the population. Later, a second dominant female-sterile mutation appeared in the monoecious population generating male plants. With time, the pair of chromosomes in which these mutations occurred stopped recombining and started accumulating mutations and repetitive elements. Recombination stopped because an individual with both mutations would become completely sterile, representing no advantage for the population. Finally, the chromosomes carrying these mutations became a pair of different sex chromosomes $[1,2]$.

Sex chromosomes are one of the most studied systems for sex determination in dioecious plants, and there are several stages of sex chromosomes already identified in many plant species $[2,3]$. Some of these species have been considered as important models for the study of sex determination and sex chromosome evolution in dioecious plants, including papaya (Carica papaya) [4]. Nevertheless, papaya is considered a trioecious species, which means that papaya populations can have individuals with either male, female or hermaphrodite flowers [5]. Natural papaya populations are dioecious, while cultivated populations are gynodioecious. In papaya, sex is determined by a recent sex chromosome system with three different sex chromosomes $\left(\mathrm{X}, \mathrm{Y}\right.$, and $\left.\mathrm{Y}^{\mathrm{h}}\right)$. Female plants are homozygous for the $\mathrm{X}$ chromosome (XX) and males and hermaphrodites are heterozygous (XY and $X Y^{\mathrm{h}}$, respectively) $[6,7]$. Between the $\mathrm{X}$ and $\mathrm{Y}^{\mathrm{h}}$ chromosomes, several differences have been identified that can explain phenotypic differences between hermaphrodite and female plants [7]. In contrast, the $\mathrm{Y}$ and $\mathrm{Y}^{\mathrm{h}}$ chromosomes have been described as highly similar $(99.60 \%)[6,8]$ and as a result, it has been challenging to explain which differences observed between male and hermaphrodite plants are responsible for their phenotypes.

Despite the genetic differences found among all these three sex chromosomes, flower development among papaya plants is very similar in its early stages. Male, female and hermaphrodite flower development start to differentiate after anthers develop in male and hermaphrodite flowers $[9,10]$. One of the main differences among the flowers is the presence of a gynoecium spear-like structure called 'pistillode' (or rudimentary pistil) in male flowers instead of a functional gynoecium, like in hermaphrodite and female flowers $[9,10]$. For this reason, it is believed that a female-sterile dominant mutation suppresses the carpel development in male flowers and that this mutation exists on the $\mathrm{Y}$ chromosome, but not on the $\mathrm{Y}^{\mathrm{h}}$ chromosome. Since the $\mathrm{Y}$ and the $\mathrm{Y}^{\mathrm{h}}$ chromosome are highly similar and most of the detected genetic differences or mutations are located on introns instead of exons [6, 8], differential gynoecium development in hermaphrodite plants and not in male plants is believed to be the result of the differential expression of a carpel development suppressor gene between sex-types. Likewise, female flowers do not have stamens, but male and hermaphrodite flowers do $[9,10]$. Therefore, a gene with male-promoting functions is believed to be located on the $\mathrm{Y}$ and the $\mathrm{Y}^{\mathrm{h}}$ chromosome.

An interesting aspect of papaya plants is that under certain environmental conditions or stimuli (e.g. high or cold temperatures, shorter day length, water stress, and terminal bud injury) male and hermaphrodite plants can switch their flower gender [11-16]. This phenomenon is known as sex-reversal and evidently affects papaya fruit production, because under undesirable environmental conditions, hermaphrodites could either reverse to male or present staminal carpellody (a condition in which the stamen resemble carpel or are 'fused' to the carpels), which results in malformed unmarketable papaya fruits $[10,17-19]$. Interestingly female plants do not suffer sexreversal, as male and hermaphrodites do. Therefore, identifying the genes responsible for the correct expression of sex or development of sex flower organs in papaya and the regulatory mechanism for the expression of those genes becomes fundamental for papaya production.

To identify the genes responsible for the correct expression of sex in papaya flowers, previous researchers have looked at the expression of homeotic genes that participate in the $\mathrm{ABC}$ model for flower development. There are few reports about differentially expressed genes among sex types and on flower development regulation by MADS-box genes in papaya [16, 20-24]. Recently, a digital transcriptome analysis of the genes located on the $\mathrm{X}$ and $\mathrm{Y}^{\mathrm{h}}$ chromosomes in papaya using high-throughput SuperSAGE technique combined with a whole-genome sequence comparison between male and hermaphrodite plants identified a Short Vegetative Phase (SVP) gene and a Monodehydroascorbate Reductase (MDAR) gene as candidates for sex determination in papaya [23, 25]. Furthermore, a recent transcriptome analysis using RNAsequencing has suggested the silencing of the carpel suppression function by epigenetic modifications (miRNAs) in male-to-hermaphrodite induced sex reversal plants 
[16]. A recent study, proposed three candidate sex-related loci, including the Short Vegetative Phase (SVP) gene and a Chromatin Assembly Factor 1 subunit A-like (CAF1AL), as responsible for regulating correct flower development in papaya, based on alternative splicing and differential expression analysis using different flower whorls [26]. Nevertheless, there is no published comparative transcriptome analysis focused on different developmental flowering stages using RNA-sequencing in papaya, including all three different sex types (including male, female and hermaphrodite flowers). Therefore, further analysis is still needed to identify the mechanisms responsible for flower development regulation in papaya, carpel development suppression in male flowers, stamen carpellody in hermaphrodite flowers and the sex reversal phenomena that occurs only in male and hermaphrodite papaya flowers.

RNA sequencing or RNA-Seq consists of the implementation of high-throughput DNA sequencing technologies for the study of transcriptomes [27, 28]. RNASeq has been described as a very powerful tool for the discovery of novel transcripts and the quantification of gene expression in model and non-model plant species, which ultimately leads to the identification of differentially expressed genes, pathways and regulatory networks that help to understand biological processes. Therefore, a differential gene expression analysis of flower buds among all three different sex types at different developmental stages during flowering can help to find differentially expressed genes associated with correct sex expression, as well as to better understand flower organ development regulation in papaya. The aim of this study is to identify genes that are differentially expressed among male, female and hermaphrodite flower buds in papaya during early and later stages of flower development using RNA-seq, and to evaluate the expression of highly differentially expressed genes by RT-qPCR, as well as to identify the putative functions for these genes based on their homology with other plant species and their expression patterns.

\section{Results}

\section{Quality control before RNA-Seq and differential expression analysis}

The transcriptome of papaya flower buds from male 'AU9', female 'AU9' and hermaphrodite 'SunUp' plants was sequenced at two different developmental stages (premeiosis: $1-6 \mathrm{~mm}$ and post-meiosis: $7-12 \mathrm{~mm}$ ) (Additional file 7: Table S1). On average, a total of $2.28 \mathrm{E}+07$ raw reads per library were obtained (Additional file 7: Table $\mathrm{S} 1)$. In general, the quality of the raw reads was classified as good by the FastQC program. Nevertheless, after trimming low-quality reads and adaptors, an average of $99.71 \%$ of these raw reads with an average length of 100 bp remained. These high-quality reads were aligned to the papaya genome. On average, a total of $83.99 \%$ reads per library were aligned uniquely to the genome, and few reads were not aligned or aligned more than once to the genome (Additional file 7: Table S1). On average, 46.08\% of the reads that aligned to the genome were assigned to exons (Additional file 7: Table S1). After normalization of the reads and before the differential expression analysis, samples were clustered, and the biological coefficient of variation was calculated as part of our analysis of quality control (Additional file 1: Figure S1). Samples clustered in three groups, one group composed of normal and teratological males of the variety 'Zhonghuang', a second group composed of female 'AU9' samples, and the third group composed by male 'AU9' and hermaphrodite 'SunUp' samples. These results reflect the existence of fewer differences found between female pre-meiosis and female postmeiosis stages, and fewer differences between male and hermaphrodite pre-meiosis stages than post-meiosis stages. No confounding batch effect was found and the calculated trend for the Biological coefficient of variation was not far from the calculated common trend (Additional file 1: Figure S1). Therefore, the analysis of differentially expressed genes was performed using the normalized expression values.

\section{Differential gene expression analysis by RNA-Seq}

From a total of 19618 analyzed genes, many were found to be differentially expressed among groups. In total, 2523 genes were differentially expressed between male and female flower buds of a size of $1-6 \mathrm{~mm}, 733$ between male and hermaphrodite flower buds of a size of 1-6 $\mathrm{mm}$ and 2165 between hermaphrodite and female flower buds of a size of 1-6 mm (Fig. 1a). Nevertheless, the number of differentially expressed genes increased among flower buds of a size of 7-12 mm. In total, 3144 genes were differentially expressed between male and female flower buds of a size of 7-12 mm, 1427 between male and hermaphrodite flower buds of a size of 7-12 $\mathrm{mm}$ and 2884 between hermaphrodite and female flower buds of a size of 7-12 mm (Fig. 1b). Only a total of 571 genes were differentially expressed between normal and teratological male (male to hermaphrodite sex reversal) pistillode (Fig. 2). In general, the number of differentially expressed genes between male and female or hermaphrodite and female flower buds was higher than the number of differentially expressed genes between male and hermaphrodite flower buds.

Since the objectives of this study were to identify candidate genes for correct sex expression between males, females and hermaphrodites, and to contribute with the understanding of flower development regulation in papaya among different sex types, only differentially expressed genes between male, female and hermaphrodite flower buds and differentially expressed between normal male 


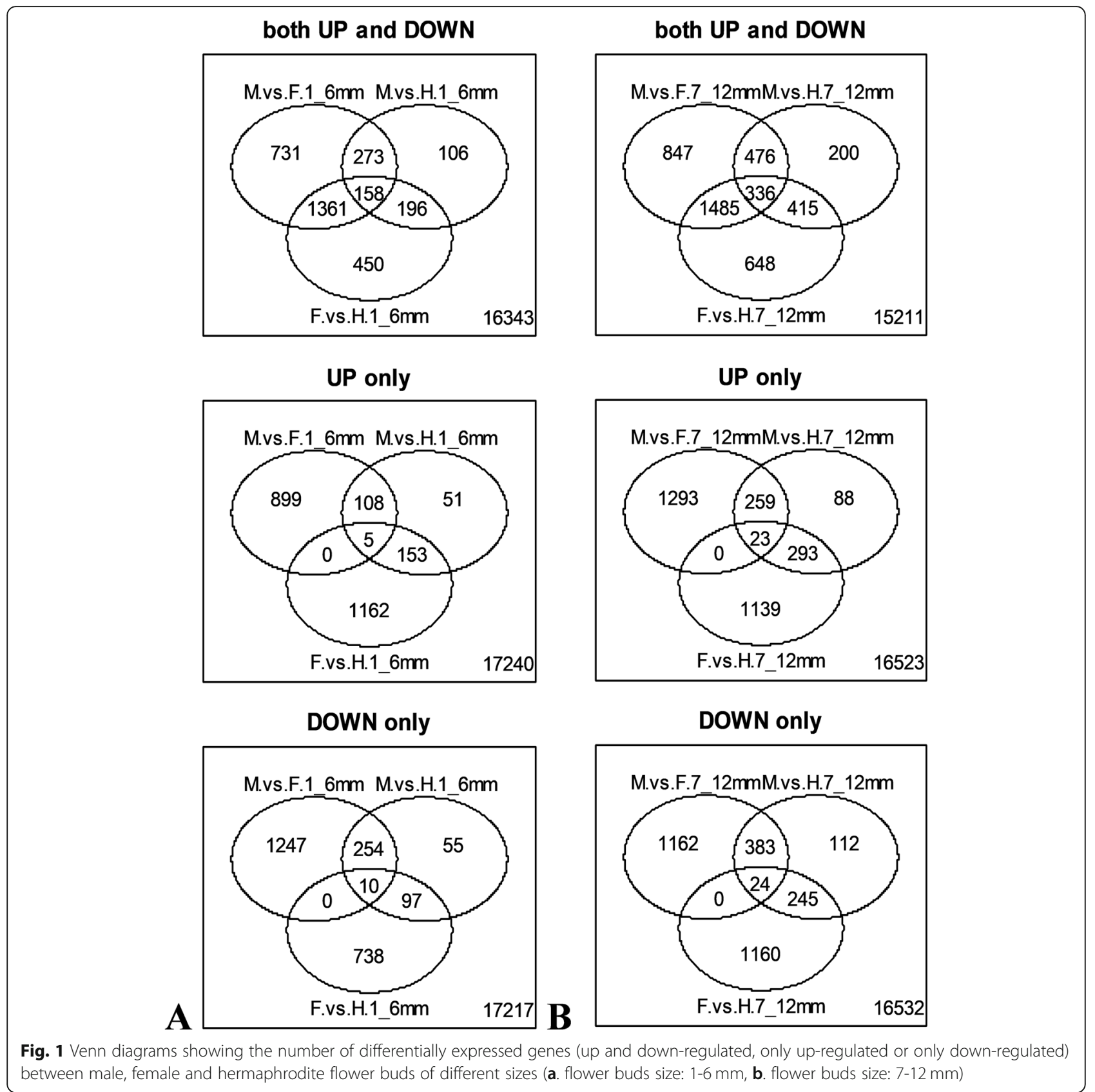

and teratological male samples were selected for further analysis (2117 genes in total). A scaled heatmap was built to compare the expression of these genes among the different samples (Fig. 3a). In the heatmap, genes that are up-regulated are shown in red, while genes that are downregulated are shown in blue. The color pattern revealed contrasting expression among samples from different sex, but less contrasting expression among samples from different stages but same-sex (Fig. 3a). Based on these colors, there is a contrast between female and male samples, in which two big groups of genes seem to be overexpressed in females but downregulated in males or overexpressed in males but downregulated in females. This clear pattern is not evident in hermaphrodite samples. In hermaphrodite samples, half of the genes upregulated in females but downregulated in males seemed upregulated, while the other half seemed downregulated and the same seemed to be the case of the genes that are upregulated in males but downregulated in females. The heatmap also reveals a small number of genes showing contrasting expression between teratological and normal male pistillode samples. A TOM (Topological Overlap Matrix) plot was also built to find out the level of complexity of the gene network involved in papaya flower development (Fig. 3b). In this plot, 


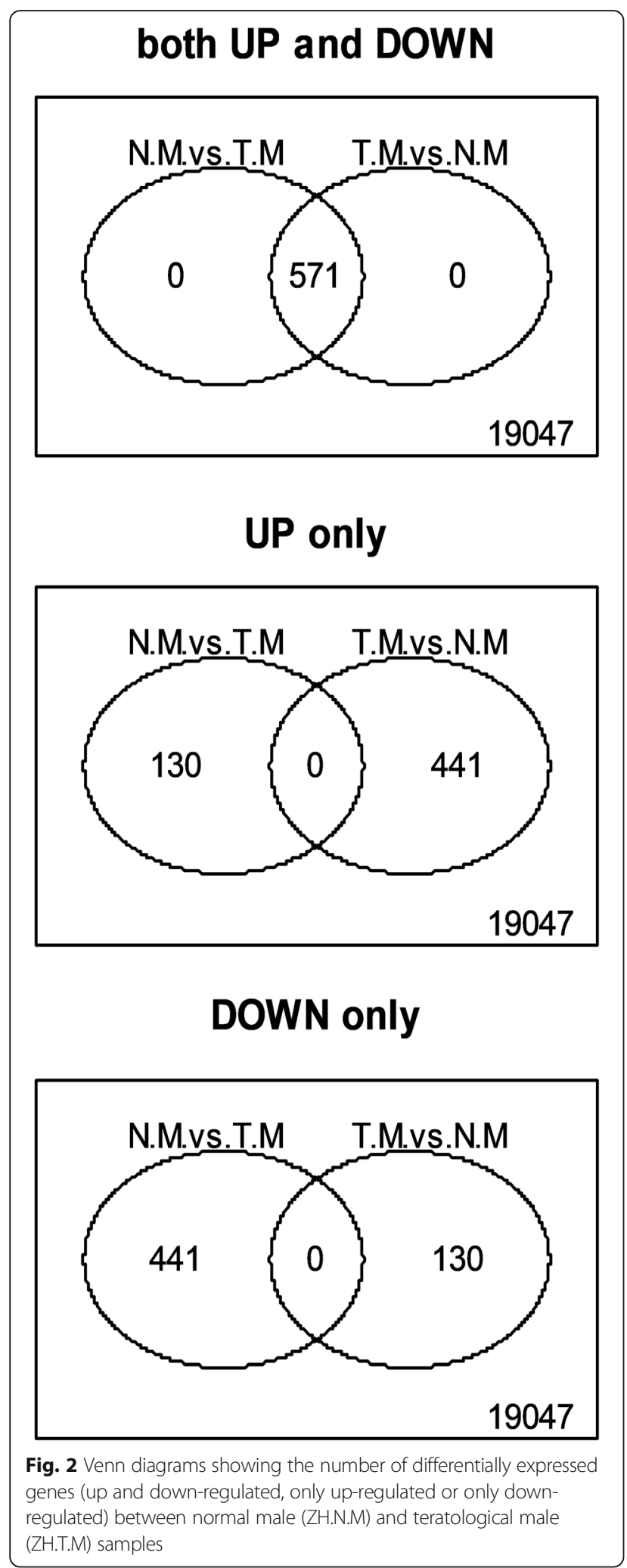

genes that have a similar expression pattern are shown in red, while genes that have no similar expression pattern are shown in yellow (Fig. 3b). The color pattern shown in this figure revealed many clusters of genes or modules that might be part of a similar pathway and a high level of complexity of the gene network for flower development.

\section{Gene Ontology analysis and over-representation results}

Gene Ontology annotations for the 2117 selected genes were analyzed and the sequences were classified into three categories according to their GO term: molecular functions (MF), biological process (BP) or cellular components (CC). In total 2081 sequences were classified in the MF category, 2632 in the BP category and 1736 in the CC category (Fig. 4). The most abundant terms for cellular components were plasma membrane, protein complexes, and nucleus (Fig. 4a). The most abundant molecular function terms were for ion binding activity, oxidoreductase activity, DNA binding, kinase activity and transmembrane transporter activity (Fig. 4b). The most abundant biological process terms were for biosynthetic processes, nitrogen metabolism, protein modification, carbohydrate metabolism, amino acid metabolism, response to stress, catabolic processes and single organism carbohydrate processes (Fig. 4c). Figure 4a, b and c also show the percentage of differentially expressed genes found for each annotation category from all individual comparisons made among the sample groups (comparisons are indicated in the figure legend).

Among biological process terms: developmental processes, reproduction and embryo development gene annotations were found (Fig. 4c). Within this last category, genes related to flower development processes and floral organ identity were found as differentially expressed (Tables 1,2 and 3) and will be further discussed. None of the genes mapped to the available papaya sex chromosome sequences $\left(\mathrm{X}, \mathrm{Y}\right.$ or $\left.\mathrm{Y}^{\mathrm{h}}\right)$, which means that the genes found in this study as differentially expressed among sex-types are not ultimately responsible for sex determination in papaya, but instead might contribute to correct sex expression or development of sex flower organs. Interestingly, the gene which showed the highest fold change between male, hermaphrodite and female flower buds was 'evm.model.supercontig_2.119' identified as a PHD-type plant homeodomain protein (PHD finger protein MALE STERILITY 1) (Tables 1 and 2).

Over-represented Gene Ontology (GO) Slim terms ( $p$ value $<0,05 ; \mathrm{FDR}<0,05$ ) were analyzed using the list of differentially expressed genes for each pairwise comparison among sample groups (Additional file 2: Figure S2, Additional file 3: Figure S3 and Additional file 4: Figure S4), to identify differences involved in flower development (common among all sex-types) and important pathways for correct sex expression. As a result, common cellular component terms identified as overrepresented were: integral and intrinsic components of membrane; microtubule and microtubule-associated 

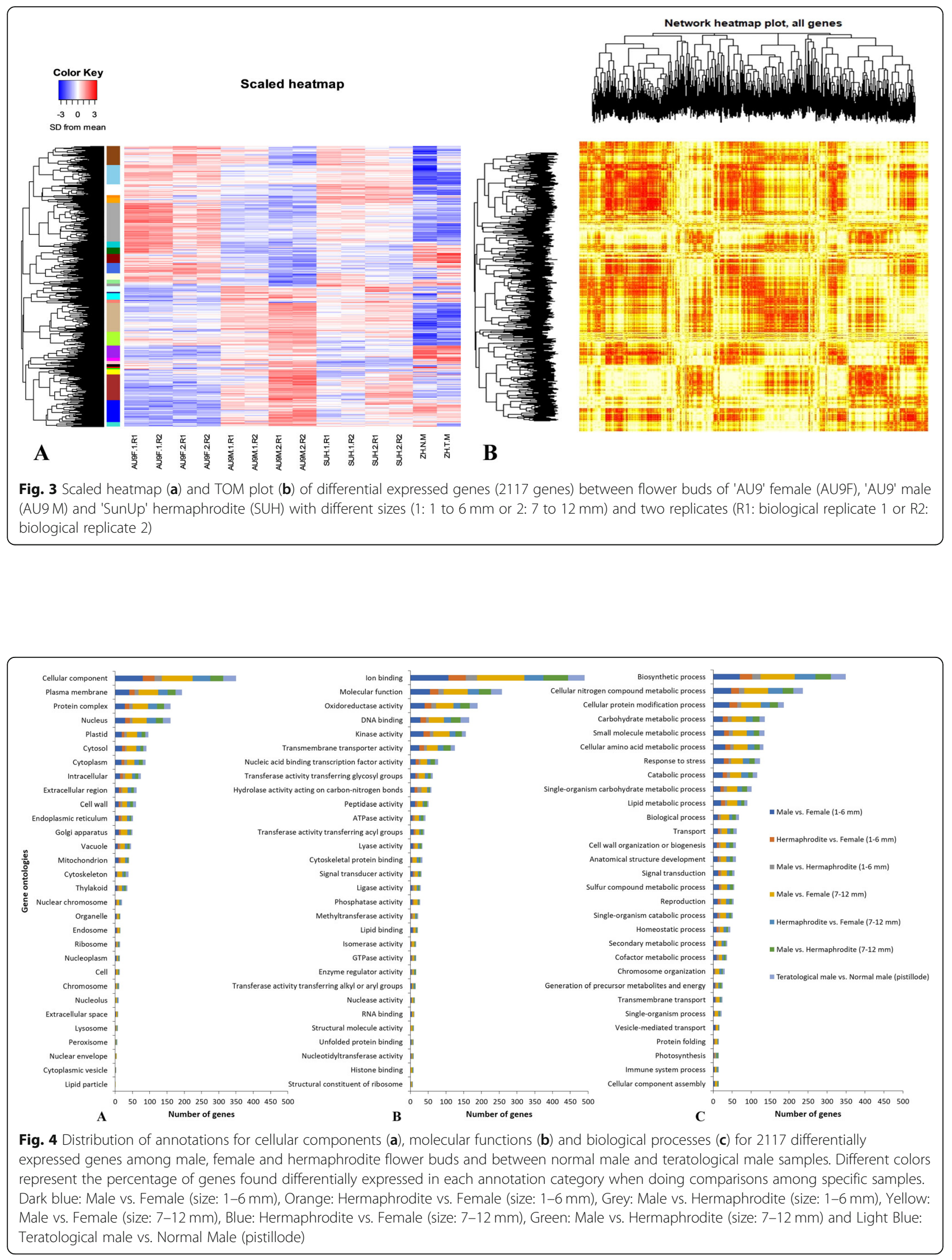
Table 1 Genes annotated for developmental processes, reproduction and/or embryo development between female, male and hermaphrodite flower buds (size 1 to $6 \mathrm{~mm}$ )

\begin{tabular}{|c|c|c|c|c|c|c|c|c|}
\hline \multirow[t]{2}{*}{ Gene ID } & \multirow[t]{2}{*}{ Description } & \multirow[t]{2}{*}{ LogCPM } & \multicolumn{2}{|c|}{ Male vs. Female } & \multicolumn{2}{|c|}{$\begin{array}{l}\text { Male vs. } \\
\text { Hermaphrodite }\end{array}$} & \multicolumn{2}{|c|}{$\begin{array}{l}\text { Hermaphrodite } \\
\text { vs. Female }\end{array}$} \\
\hline & & & $\mathrm{FC}^{*}$ & $\mathrm{FDR}^{*}$ & $\mathrm{FC}^{*}$ & $\mathrm{FDR}^{*}$ & $\mathrm{FC}^{*}$ & $\mathrm{FDR}^{*}$ \\
\hline evm.model.supercontig_2.119 & PHD Finger MALE STERILITY 1 & 5.87 & 2835.03 & 2.99E-02 & 294.20 & $1.01 \mathrm{E}-01$ & 9.64 & $9.69 \mathrm{E}-01$ \\
\hline evm.model.supercontig_157.32 & Glucan endo-1,3-beta-glucosidase-like & 5.20 & 30.13 & 4.50E-02 & 19.58 & 1.69E-01 & 1.54 & $9.79 \mathrm{E}-\mathrm{C}$ \\
\hline evm.model.supercontig_87.13 & PHD Finger MALE MEIOCYTE DEATH 1 & 0.11 & 21.62 & $2.82 \mathrm{E}-02$ & 1.32 & $1.00 \mathrm{E}+00$ & 16.44 & $6.83 \mathrm{E}-\mathrm{C}$ \\
\hline evm.model.supercontig_85.48 & Probable kinase & 1.80 & 9.14 & $1.72 \mathrm{E}-02$ & 1.92 & $1.00 \mathrm{E}+00$ & 4.77 & $1.68 \mathrm{E}-\mathrm{C}$ \\
\hline evm.model.supercontig_50.85 & Glycerol-3-phosphate acyltransferase 1 & 5.70 & 6.16 & 4.05E-02 & 4.31 & 2.07E-01 & 1.43 & $8.91 \mathrm{E}-$ \\
\hline evm.model.supercontig_1525.1 & Abscisic acid 8-hydroxylase 1 & 5.22 & 5.32 & $1.34 \mathrm{E}-04$ & 3.72 & $2.57 \mathrm{E}-03$ & 1.43 & $3.48 \mathrm{E}-0$ \\
\hline evm.model.supercontig_6.104 & $\begin{array}{l}\text { Steroid 22-alpha-hydroxylase/ cytochrome } \\
\text { P450 90B1-like }\end{array}$ & 3.97 & 4.73 & $2.98 \mathrm{E}-04$ & 1.24 & $1.00 E+00$ & 3.81 & $3.18 \mathrm{E}$ \\
\hline evm.model.supercontig_75.17 & E3 ubiquitin-protein ligase XBAT32/33 & 4.14 & 3.49 & $1.02 \mathrm{E}-03$ & 2.23 & 1.49E-01 & 1.56 & $5.40 \mathrm{E}-$ \\
\hline evm.model.supercontig_26.241 & Cytochrome P450 83B1 (CYP83B1) & 4.92 & 2.54 & 2.93E-02 & 1.12 & $1.00 \mathrm{E}+00$ & 2.26 & $6.18 \mathrm{E}-$ \\
\hline evm.model.supercontig_427.3 & Protein of unknown function (DUF642) & 6.77 & 2.40 & $1.64 \mathrm{E}-04$ & -1.41 & 3.79E-01 & 3.38 & 2.66 \\
\hline evm.model.supercontig_27.166 & $\begin{array}{l}\text { G-type lectin S-receptor-like serine threonine- } \\
\text { kinase RKS1 }\end{array}$ & 4.48 & 2.09 & 4.30E-02 & 1.72 & 4.07E-01 & 1.21 & $8.95 \mathrm{E}$ \\
\hline evm.model.supercontig_6.12 & $\begin{array}{l}\text { Pentatricopeptide repeat-containing } p \\
\text { chloroplastic }\end{array}$ & 5.38 & 2.02 & $3.09 \mathrm{E}-03$ & 1.57 & $1.58 \mathrm{E}-01$ & 1.29 & 5 \\
\hline evm.model.supercontig_26.316 & Floral homeotic protein PISTILLATA & 6.99 & 1.83 & 4.97E-03 & -1.17 & $1.00 \mathrm{E}+00$ & 2.14 & $1.44 \mathrm{E}-$ \\
\hline evm.model.supercontig_16.181 & Condensin-2 complex subunit D3 (NCAPD3) & 4.54 & 1.44 & $5.41 \mathrm{E}-01$ & -1.76 & $3.23 \mathrm{E}-01$ & 2.53 & $5.09 \mathrm{E}-$ \\
\hline evm.model.supercontig_4 & Transcription factor M & 1.68 & 1.16 & 9.91E-01 & 8.43 & $6.06 \mathrm{E}-02$ & -7.25 & 4.32 \\
\hline evm.model.supercontig_59.35 & Ammonium transporter 1 member 2 & 7.05 & -1.42 & 1.95E-01 & 1.45 & $3.10 \mathrm{E}-01$ & -2.06 & $7.87 \mathrm{E}$ \\
\hline evm.model.supercontig_13.86 & Tetraspanin-11 related & 2.43 & -1.95 & $6.84 \mathrm{E}-01$ & -7.74 & $1.92 \mathrm{E}-02$ & 3.96 & $1.02 \mathrm{E}$ \\
\hline evm.model.supercontig_12.194 & Scarecrow-like transcription factor PAT1 & 7.00 & -2.09 & 2.49E-04 & -1.62 & 8.51E-02 & -1.29 & 4.66 \\
\hline evm.model.supercontig_70.45 & Potassium transporter 4 & 7.87 & -2.10 & $2.55 \mathrm{E}-03$ & -1.21 & $1.00 \mathrm{E}+00$ & -1.73 & $1.72 \mathrm{E}$ \\
\hline evm.model.supercontig_33.178 & Calmodulin & 9.88 & -2.12 & 7.14E-04 & -1.97 & $5.38 \mathrm{E}-03$ & -1.07 & $9.75 \mathrm{E}$ \\
\hline evm.model.supercontig_8.181 & Sterol 3-beta-glucosyltransferase UGT80A2 & 7.17 & -2.14 & $6.60 \mathrm{E}-04$ & -1.50 & 2.37E-01 & -1.43 & 1.93E- \\
\hline evm.model.supercontig_18.43 & bZIP transcription factor 53 & 6.73 & -2.15 & $1.32 \mathrm{E}-04$ & -1.54 & $1.42 \mathrm{E}-01$ & -1.40 & 2.19 \\
\hline evm.model.supercontig_27.172 & $\begin{array}{l}\text { G-type lectin S-receptor-like serine threonine- } \\
\text { kinase B120 }\end{array}$ & 4.56 & -2.20 & $1.84 \mathrm{E}-02$ & -1.21 & $1.00 \mathrm{E}+00$ & -1.82 & $1.27 \mathrm{E}$ \\
\hline evm.model.supercontig_52.111 & VAN3-binding/Auxin canalisation (Auxin canalis) & 5.61 & -2.22 & 5.19E-04 & -1.64 & $6.37 \mathrm{E}-02$ & -1.35 & $2.98 \mathrm{E}-$ \\
\hline evm.model.supercontig_132.29 & $\begin{array}{l}\text { E3 ubiquitin- ligase COP1-like/ Protein suppressor } \\
\text { of PHYA-105 } 1\end{array}$ & 4.30 & -2.22 & $3.88 \mathrm{E}-02$ & 1.09 & $1.00 \mathrm{E}+00$ & -2.43 & $2.14 \mathrm{E}$ \\
\hline evm.model.supercontig_6.349 & Thioredoxin reductase 2 -like & 5.96 & -2.50 & $8.72 \mathrm{E}-05$ & -1.67 & $3.59 \mathrm{E}-02$ & -1.50 & 7.78E- \\
\hline evm.model.supercontig_18.81 & Transcription factor UPBEAT1 & 2.86 & -3.24 & $1.12 \mathrm{E}-01$ & -5.37 & $2.85 \mathrm{E}-02$ & 1.66 & 7.36E \\
\hline evm.model.supercontig_25.116 & $\begin{array}{l}\text { AP2-like ethylene-responsive transcription } \\
\text { factor AlL1 }\end{array}$ & 2.96 & -3.71 & 4.92E-02 & -1.19 & $1.00 \mathrm{E}+00$ & -3.11 & $1.24 \mathrm{E}$ \\
\hline evm.model.supercontig_160.4 & Receptor kinase HAIKU2 & 9.55 & -3.71 & 9.38E-04 & -1.95 & $1.01 \mathrm{E}-01$ & -1.90 & $5.32 \mathrm{E}-$ \\
\hline evm.model.supercontig_49.19 & Abscisic acid 8 -hydroxylase 4 & 3.51 & -4.53 & $5.74 \mathrm{E}-03$ & -6.17 & 7.33E-03 & 1.36 & $8.60 \mathrm{E}$ \\
\hline evm.model.supercontig_444.5 & Beta-glucosidase 27-like & 1.15 & -10.02 & $2.50 \mathrm{E}-02$ & -5.43 & $3.00 \mathrm{E}-01$ & -1.85 & $8.20 \mathrm{E}$ \\
\hline evm.model.supercontig_189.36 & Beta-glucosidase 32-like & 2.34 & -17.19 & 7.54E-03 & -3.89 & $3.65 \mathrm{E}-01$ & -4.42 & $1.15 \mathrm{E}$ \\
\hline evm.model.supercontig_1.155 & $\begin{array}{l}\text { Cytochrome P450 85A-like/ Brassinosteroid-6- } \\
\text { oxidase } 2 \text { (CYP85A2, BR6OX2) }\end{array}$ & -0.77 & -60.77 & 3.13E-02 & -8.79 & 7.56E-01 & -6.91 & $2.89 \mathrm{E}$ \\
\hline
\end{tabular}

${ }^{a}$ Differentially expressed genes parameters: Fold-Change $(\mathrm{FC})>2$ or $<-2$ and a False Discovery Rate $(\mathrm{FDR})<0.05$

complex; nucleus; polymeric cytoskeletal fiber; supramolecular complex and fiber; and supramolecular complex, fiber and polymer (Additional file 2: Figure S2, shown in blue). Nevertheless, highly over-represented cellular component terms were: chloroplast thylakoid membrane; plant-type vacuole and plastoglobuli 
Table 2 Genes annotated for developmental processes, reproduction and/or embryo development between female, male and hermaphrodite flower buds (size 7 to $12 \mathrm{~mm}$ )

\begin{tabular}{|c|c|c|c|c|c|c|c|c|}
\hline \multirow[t]{2}{*}{ Gene ID } & \multirow[t]{2}{*}{ Description } & \multirow[t]{2}{*}{ LogCPM } & \multicolumn{2}{|c|}{$\begin{array}{l}\text { Male } \\
\text { vs. Female }\end{array}$} & \multicolumn{2}{|c|}{$\begin{array}{l}\text { Male vs. } \\
\text { Hermaphrodite }\end{array}$} & \multicolumn{2}{|c|}{$\begin{array}{l}\text { Hermaphrodite } \\
\text { vs. Female }\end{array}$} \\
\hline & & & $\mathrm{FC}^{*}$ & $\mathrm{FDR}^{*}$ & $\overline{F C^{*}}$ & $\mathrm{FDR}^{*}$ & $\overline{F C^{*}}$ & $\mathrm{FDR}^{*}$ \\
\hline evm.model.supercontig_2.119 & PHD Finger MALE STERILITY 1 & 5.87 & 3350.22 & 7.72E-03 & 1.07 & $1.00 \mathrm{E}+00$ & 3118.56 & $8.87 \mathrm{E}-03$ \\
\hline evm.model.supercontig_157.32 & Glucan endo-1,3-beta-glucosidase-like & 5.20 & 75.59 & $1.38 \mathrm{E}-02$ & 2.31 & 7.94E-01 & 32.78 & 3.33E-02 \\
\hline evm.model.supercontig_72.35 & Egg cell secreted protein-like & -0.25 & 56.37 & $2.66 \mathrm{E}-02$ & 3.13 & $6.71 \mathrm{E}-01$ & 18.04 & 1.04E-01 \\
\hline evm.model.supercontig_3.299 & Cellulose synthase-like protein D1 & -0.33 & 23.88 & 4.03E-02 & 1.33 & $1.00 \mathrm{E}+00$ & 17.98 & $6.28 \mathrm{E}-02$ \\
\hline evm.model.supercontig_182.26 & Cytokinin dehydrogenase 6 & 0.95 & 17.90 & 8.92E-03 & 8.14 & $9.47 \mathrm{E}-02$ & 2.20 & $7.73 \mathrm{E}-01$ \\
\hline evm.model.supercontig_14.52 & Actin cross-linking (DUF569) & 0.94 & 13.07 & $1.28 \mathrm{E}-02$ & 6.39 & $1.35 \mathrm{E}-01$ & 2.05 & 7.61E-01 \\
\hline evm.model.supercontig_50.85 & Glycerol-3-phosphate acyltransferase 1 & 5.70 & 10.39 & 1.30E-02 & -1.20 & $1.00 \mathrm{E}+00$ & 12.49 & $1.04 \mathrm{E}-02$ \\
\hline evm.model.supercontig_1525.1 & Abscisic acid 8-hydroxylase 1 & 5.22 & 9.79 & $2.90 \mathrm{E}-05$ & -1.67 & $1.26 \mathrm{E}-01$ & 16.31 & $1.38 \mathrm{E}-05$ \\
\hline evm.model.supercontig_85.48 & Probable kinase & 1.80 & 8.78 & 1.07E-02 & 1.21 & $1.00 \mathrm{E}+00$ & 7.24 & 2.39E-02 \\
\hline evm.model.supercontig_11.32 & $\begin{array}{l}\text { Calmodulin-binding receptor-like cytoplasmic } \\
\text { kinase } 2\end{array}$ & 4.72 & 4.03 & $2.88 \mathrm{E}-05$ & 1.22 & 9.77E-01 & 3.31 & 8.43E-05 \\
\hline evm.model.supercontig_6.104 & $\begin{array}{l}\text { Steroid 22-alpha-hydroxylase /cytochrome P450 } \\
\text { 90B1-like }\end{array}$ & 3.97 & 3.31 & 5.19E-03 & 1.19 & $1.00 \mathrm{E}+00$ & 2.78 & 2.40E-02 \\
\hline evm.model.supercontig_27.166 & $\begin{array}{l}\text { G-type lectin S-receptor-like serine threonine- } \\
\text { kinase RKS1 }\end{array}$ & 4.48 & 3.22 & 2.73E-04 & 1.88 & $1.41 \mathrm{E}-01$ & 1.71 & $1.98 \mathrm{E}-01$ \\
\hline evm.model.supercontig_89.64 & Homeobox-leucine zipper HAT4-like & 5.04 & 3.20 & $1.94 \mathrm{E}-05$ & 1.67 & $1.14 \mathrm{E}-01$ & 1.92 & $1.64 \mathrm{E}-02$ \\
\hline evm.model.supercontig_75.17 & E3 ubiquitin-protein ligase XBAT32/33 & 4.14 & 2.97 & 4.10E-03 & -1.35 & $9.01 \mathrm{E}-01$ & 4.02 & 2.85E-04 \\
\hline evm.model.supercontig_26.316 & Floral homeotic protein PISTILLATA & 6.99 & 2.80 & 1.39E-04 & 1.30 & $5.57 \mathrm{E}-01$ & 2.16 & $1.03 \mathrm{E}-03$ \\
\hline evm.model.supercontig_26.81 & Protein GIGANTEA & 7.41 & 2.51 & $3.21 \mathrm{E}-03$ & 1.48 & 2.67E-01 & 1.70 & $6.18 \mathrm{E}-02$ \\
\hline evm.model.supercontig_26.82 & GIGANTEA-like & 9.07 & 2.50 & $1.54 \mathrm{E}-04$ & 1.40 & $3.04 \mathrm{E}-01$ & 1.78 & 7.36E-03 \\
\hline evm.model.supercontig_23.39 & $\begin{array}{l}\text { UDP-sugar transporter/Solute carrier family } 35 \\
\text { (SLC35D) }\end{array}$ & 4.45 & 2.38 & $9.96 \mathrm{E}-03$ & 1.60 & 4.23E-01 & 1.49 & 4.23E-01 \\
\hline evm.model.supercontig_44.60 & Transcription factor MYB4-like & 1.68 & 2.22 & 5.37E-01 & 8.95 & $2.66 \mathrm{E}-02$ & -4.03 & $1.88 \mathrm{E}-01$ \\
\hline evm. TU.contig_29408.2 & Metal-nicotianamine transporter YSL3 & 6.63 & 2.11 & $1.14 \mathrm{E}-04$ & 1.47 & $1.46 \mathrm{E}-01$ & 1.43 & $1.33 \mathrm{E}-01$ \\
\hline evm.model.supercontig_87.13 & PHD Finger MALE MEIOCYTE DEATH 1 & 0.11 & 1.98 & 8.37E-01 & -8.14 & $1.53 \mathrm{E}-01$ & 16.08 & $2.38 \mathrm{E}-02$ \\
\hline evm.model.supercontig_1.61 & Serine threonine-kinase STY46 & 4.67 & 1.96 & 3.69E-02 & 1.16 & $1.00 \mathrm{E}+00$ & 1.68 & $1.54 \mathrm{E}-01$ \\
\hline evm.model.supercontig_20.162 & Expansin-like A2 & 6.35 & 1.38 & $1.94 \mathrm{E}-01$ & -1.47 & $1.35 \mathrm{E}-01$ & 2.03 & $7.58 \mathrm{E}-04$ \\
\hline evm.model.supercontig_13.86 & Tetraspanin-11 related & 2.43 & -1.01 & $\begin{array}{l}1.00 \mathrm{E}+ \\
00\end{array}$ & -7.96 & 7.60E-03 & 7.87 & 4.14E-03 \\
\hline evm. TU.contig_30608.1 & CRABS CLAW/ HMG-box domain (HMG box 2) & 7.20 & -1.50 & 9.34E-02 & 1.50 & $1.80 \mathrm{E}-01$ & -2.25 & $2.14 \mathrm{E}-03$ \\
\hline evm.model.supercontig_97.108 & $\begin{array}{l}\text { Epidermis-specific secreted glyco EP1-like/ } \\
\text { COMITIN }\end{array}$ & 4.66 & -1.58 & $2.26 \mathrm{E}-01$ & 1.71 & $2.10 \mathrm{E}-01$ & -2.71 & $8.29 \mathrm{E}-04$ \\
\hline evm.model.supercontig_4.62 & Callose synthase 10 & 4.99 & -1.59 & $3.28 \mathrm{E}-01$ & -3.62 & $1.35 \mathrm{E}-02$ & 2.28 & $5.43 \mathrm{E}-02$ \\
\hline evm.model.supercontig_27.172 & $\begin{array}{l}\text { G-type lectin S-receptor-like serine threonine- } \\
\text { kinase B120 }\end{array}$ & 4.56 & -1.76 & $1.26 \mathrm{E}-01$ & 1.21 & $1.00 \mathrm{E}+00$ & -2.13 & 2.44E-02 \\
\hline evm.model.supercontig_16.181 & Condensin-2 complex subunit D3 (NCAPD3) & 4.54 & -1.88 & 8.63E-02 & -2.97 & $2.11 \mathrm{E}-03$ & 1.58 & 2.84E-01 \\
\hline evm.model.supercontig_115.9 & Transcription factor HY5 & 5.54 & -1.92 & 1.10E-03 & 1.05 & $1.00 \mathrm{E}+00$ & -2.01 & 4.61E-04 \\
\hline evm.model.supercontig_75.60 & Protein kinase PINOID 2 & 5.61 & -2.09 & $1.61 \mathrm{E}-04$ & 1.25 & 7.16E-01 & -2.61 & 1.97E-05 \\
\hline evm.model.supercontig_12.194 & Scarecrow-like transcription factor PAT1 & 7.00 & -2.13 & 1.63E-04 & -2.10 & 5.92E-04 & -1.01 & 9.96E-01 \\
\hline evm.model.supercontig_132.29 & $\begin{array}{l}\text { E3 ubiquitin- ligase COP1-like/ Protein } \\
\text { suppressor of PHYA-105 } 1\end{array}$ & 4.30 & -2.18 & 4.00E-02 & -1.06 & $1.00 E+00$ & -2.06 & $6.58 \mathrm{E}-02$ \\
\hline evm.model.supercontig_621.3 & Major facilitator protein/ Spinster homolog 3 & 4.27 & -2.31 & $2.98 \mathrm{E}-02$ & -1.57 & 5.77E-01 & -1.47 & 5.17E-01 \\
\hline evm.model.supercontig_84.92 & Histone $\mathrm{H} 3$ isoform 1 & 6.36 & -2.41 & $1.58 \mathrm{E}-04$ & -1.08 & $1.00 \mathrm{E}+00$ & -2.24 & 3.17E-04 \\
\hline evm.model.supercontig_233.1 & AP2-like ethylene-responsive transcription & 5.82 & -2.54 & 4.40E-05 & -2.28 & $2.83 \mathrm{E}-04$ & -1.11 & 8.64E-01 \\
\hline
\end{tabular}


Table 2 Genes annotated for developmental processes, reproduction and/or embryo development between female, male and hermaphrodite flower buds (size 7 to $12 \mathrm{~mm}$ ) (Continued)

\begin{tabular}{|c|c|c|c|c|c|c|c|c|}
\hline \multirow[t]{2}{*}{ Gene ID } & \multirow[t]{2}{*}{ Description } & \multirow[t]{2}{*}{ LogCPM } & \multicolumn{2}{|c|}{$\begin{array}{l}\text { Male } \\
\text { vs. Female }\end{array}$} & \multicolumn{2}{|c|}{$\begin{array}{l}\text { Male vs. } \\
\text { Hermaphrodite }\end{array}$} & \multicolumn{2}{|c|}{$\begin{array}{l}\text { Hermaphrodite } \\
\text { vs. Female }\end{array}$} \\
\hline & & & $\overline{F C^{*}}$ & $\mathrm{FDR}^{*}$ & $\overline{\mathrm{FC}}{ }^{*}$ & $\mathrm{FDR}^{*}$ & $\mathrm{FC}^{*}$ & $\mathrm{FDR}^{*}$ \\
\hline evm.model.supercontig_84.93 & Histone $\mathrm{H} 3$ isoform 2 & 5.65 & -2.85 & 8.95E-05 & 1.07 & $1.00 \mathrm{E}+00$ & -3.04 & 7.75E-05 \\
\hline evm.model.supercontig_97.109 & COMITIN & 2.68 & -2.92 & 2.89E-01 & -6.83 & 2.47E-02 & 2.34 & $3.89 \mathrm{E}-0$ \\
\hline evm.model.supercontig_200.13 & Cyclin-D3-1 & 4.91 & -3.19 & $1.38 \mathrm{E}-04$ & -2.75 & $9.81 \mathrm{E}-04$ & -1.16 & $8.45 \mathrm{E}-0$ \\
\hline evm.model.supercontig_3.468 & Kinesin-like protein NACK1 & 4.73 & -3.35 & 1.45E-04 & -2.25 & $1.57 \mathrm{E}-02$ & -1.49 & $3.23 \mathrm{E}-0$ \\
\hline evm.model.supercontig_53.153 & Shugoshin-1-like/ Shugosin C terminus & 3.12 & -3.63 & 3.33E-02 & -3.09 & $1.28 \mathrm{E}-01$ & -1.17 & $9.58 \mathrm{E}-0$ \\
\hline evm.model.supercontig_81.9 & Kinesin family member C1 (KIFC1) & 5.45 & -3.88 & $8.46 \mathrm{E}-05$ & -1.83 & 2.73E-02 & -2.12 & $3.39 \mathrm{E}-0$ \\
\hline evm.model.supercontig_125.26 & DNA topoisomerase 2 (TOP2) & 5.65 & -3.93 & 1.03E-04 & -1.80 & $3.67 \mathrm{E}-02$ & -2.18 & 3.47E-03 \\
\hline evm.model.supercontig_21.170 & WUSCHEL-related homeobox 4 & 4.45 & -4.38 & $1.31 \mathrm{E}-04$ & -2.32 & 3.59E-02 & -1.89 & 9.26E-02 \\
\hline evm.model.supercontig_151.45 & 1,4-beta-D-xylan synthase & 5.73 & -4.90 & $6.66 \mathrm{E}-05$ & -2.18 & $1.27 \mathrm{E}-02$ & -2.25 & 4.04E \\
\hline evm.model.supercontig_160.4 & Receptor kinase HAIKU2 & 9.55 & -5.04 & $2.34 \mathrm{E}-04$ & -2.55 & $1.29 \mathrm{E}-02$ & -1.98 & $3.24 \mathrm{E}-02$ \\
\hline evm.model.supercontig_129.70 & $\begin{array}{l}\text { AP2-like ethylene-responsive transcription factor } \\
\text { ANT }\end{array}$ & 4.92 & -5.60 & 3.90E-04 & -2.22 & $6.02 \mathrm{E}-02$ & -2.52 & $1.30 \mathrm{E}-0$ \\
\hline evm.model.supercontig_18.81 & Transcription factor UPBEAT1 & 2.86 & -5.65 & 7.56E-03 & -4.53 & 4.53E-02 & -1.25 & 9.29E-01 \\
\hline evm.model.supercontig_160.33 & $\begin{array}{l}\text { AP2-like ethylene-responsive transcription factor } \\
\text { ANT }\end{array}$ & 5.37 & -5.94 & $1.66 \mathrm{E}-05$ & -1.42 & 3.44E-01 & -4.19 & $6.01 \mathrm{E}-05$ \\
\hline evm.model.supercontig_52.111 & VAN3-binding/Auxin canalisation (Auxin canalis) & 5.61 & -6.01 & 4.68E-06 & -1.96 & 4.19E-03 & -3.06 & $6.53 \mathrm{E}-$ \\
\hline evm.model.supercontig_25.116 & $\begin{array}{l}\text { AP2-like ethylene-responsive transcription factor } \\
\text { AlL1 }\end{array}$ & 2.96 & -6.82 & $1.68 \mathrm{E}-03$ & 1.67 & $9.08 \mathrm{E}-01$ & -11.43 & 1.37E-04 \\
\hline evm.model.supercontig_26.241 & Cytochrome P450 83B1 (CYP83B1) & 4.92 & -8.81 & 4.39E-04 & -8.42 & $1.36 \mathrm{E}-03$ & -1.05 & 9.87E-01 \\
\hline evm.model.supercontig_444.5 & Beta-glucosidase 27-like & 1.15 & -20.46 & $9.46 \mathrm{E}-03$ & -6.73 & $2.02 \mathrm{E}-01$ & -3.04 & 3.87E- \\
\hline evm.model.supercontig_1.155 & $\begin{array}{l}\text { Cytochrome P450 85A-like/ Brassinosteroid-6- } \\
\text { oxidase } 2 \\
\text { (CYP85A2, BR6OX2) }\end{array}$ & -0.77 & -104.77 & $1.73 \mathrm{E}-02$ & -8.57 & $6.54 \mathrm{E}-01$ & -12.22 & 7.15E-02 \\
\hline
\end{tabular}

${ }^{a}$ Differentially expressed genes parameters: Fold-Change $(\mathrm{FC})>2$ or $<-2$ and a False Discovery Rate $(\mathrm{FDR})<0.05$

(Additional file 2: Figure S2, showed in red). Common molecular function terms identified as over-represented were: transmembrane transporter activity; ATPase activity; catalytic activity; lyase activity; oxidoreductase activity; and transporter activity (Additional file 3: Figure S3, showed in blue). Highly over-represented molecular function terms were: amide transmembrane transporter activity; ATP-dependent microtubule motor activity, peptide, and oligopeptide transmembrane transporter activity (Additional file 3: Figure S3, showed in red). Common biological process terms identified as overrepresented were: microtubule-based movement; response to oxygen-containing compounds; and small molecule metabolic process (Additonal file 4: Figure S4, showed in blue). Highly over-represented biological process terms were: inorganic anion transmembrane transport; jasmonate mediated signaling pathway; regulation of defense response, response to stimulus, response to stress, signal transduction, heat and wounding (Additional file 4: Figure S4, showed in red). These results suggest that differentially expressed genes that participate in processes related to response to stress conditions, response to oxygen-containing compounds and external stimuli, as well, as molecular functions related to transmembrane transport and oxidoreductase activity might be considered important for flower development and correct sex expression in papaya.

\section{RT-qPCR expression analysis of $\mathrm{CpMS1}$}

Since the 'evm.model.supercontig_2.119' or CpMS1 gene presented extremely highest Fold Change (FC) among sex types during early and late flower developmental stages, the expression of genes that are reported to regulate MALE STERILITY 1 expression in model plants was also examined (Table 4), CpMS1 over-expression was validated by qPCR in male flower buds and other characteristics of this gene were explored.

The relative expression or Fold Change (FC) of the PHD finger protein MALE STERILITY 1 was obtained by qPCR and compared among sex-types. Interestingly, this male sterility gene (CpMS1) did not amplify in the leaf tissue samples of female, hermaphrodite or male plants; which suggests that its expression is specific for flowers (tissue-specific expression). Furthermore, this 
Table 3 Genes annotated for developmental processes, reproduction and/or embryo development between normal and teratological male

\begin{tabular}{|c|c|c|c|c|}
\hline \multirow[t]{2}{*}{ Gene ID } & \multirow[t]{2}{*}{ Description } & \multirow[t]{2}{*}{ LogCPM } & \multicolumn{2}{|c|}{ Normal male vs. Teratological male } \\
\hline & & & $\mathrm{FC}^{*}$ & $\mathrm{FDR}^{*}$ \\
\hline evm.model.supercontig_6.104 & Steroid 22-alpha-hydroxylase /cytochrome P450 90B1-like & 3.97 & 5.91 & $8.19 \mathrm{E}-03$ \\
\hline evm.model.supercontig_1525.1 & Abscisic acid 8-hydroxylase 1 & 5.22 & 4.89 & 1.30E-02 \\
\hline evm.model.supercontig_427.3 & Protein of unknown function (DUF642) & 6.77 & 4.33 & $3.66 \mathrm{E}-04$ \\
\hline evm.model.supercontig_26.316 & Floral homeotic protein PISTILLATA & 6.99 & 3.62 & $1.79 \mathrm{E}-02$ \\
\hline evm.model.supercontig_70.45 & Potassium transporter 4 & 7.87 & 3.45 & 4.35E-03 \\
\hline evm. TU.contig_30608.1 & CRABS CLAW/ HMG-box domain (HMG box 2) & 7.20 & 3.04 & $8.59 \mathrm{E}-03$ \\
\hline evm.model.supercontig_7.3 & Auxin response factor 3 & 5.25 & 2.79 & $6.33 \mathrm{E}-03$ \\
\hline evm.model.supercontig_2.240 & DELLA GAIP-B-like & 7.10 & -2.16 & 4.21E-02 \\
\hline evm.model.supercontig_55.116 & Floral homeotic protein APETALA 2 & 6.93 & -2.41 & $1.21 \mathrm{E}-02$ \\
\hline evm.model.supercontig_233.1 & AP2-like ethylene-responsive transcription factor AlL5 & 5.82 & -2.63 & $2.89 \mathrm{E}-03$ \\
\hline evm.model.supercontig_157.48 & DNA repair protein RAD50 & 5.43 & -2.76 & 4.12E-02 \\
\hline evm.model.supercontig_81.9 & Kinesin family member C1 (KIFC1) & 5.45 & -2.83 & $2.85 \mathrm{E}-02$ \\
\hline evm.model.supercontig_200.13 & Cyclin-D3-1 & 4.91 & -2.85 & 4.18E-02 \\
\hline evm.model.supercontig_52.111 & VAN3-binding/Auxin canalisation (Auxin canalis) & 5.61 & -3.44 & $2.52 \mathrm{E}-03$ \\
\hline evm.model.supercontig_6.188 & 3-epi-6-deoxocathasterone 23-monooxygenase (CYP90C1, ROT3) & 7.20 & -3.90 & 1.30E-03 \\
\hline evm.model.supercontig_3.468 & Kinesin-like protein NACK1 & 4.73 & -4.49 & 6.47E-03 \\
\hline evm.model.supercontig_129.70 & AP2-like ethylene-responsive transcription factor ANT & 4.92 & -4.92 & 1.97E-02 \\
\hline evm.model.supercontig_127.38 & BTB POZ domain-containing NPY1 & 5.96 & -5.36 & $1.83 \mathrm{E}-03$ \\
\hline evm.model.supercontig_151.45 & 1,4-beta-D-xylan synthase & 5.73 & -5.86 & $2.76 \mathrm{E}-03$ \\
\hline evm.model.supercontig_125.26 & DNA topoisomerase 2 (TOP2) & 5.65 & -6.34 & $2.24 \mathrm{E}-03$ \\
\hline evm.model.supercontig_84.93 & Histone $\mathrm{H} 3$ & 5.65 & -6.46 & $1.93 \mathrm{E}-03$ \\
\hline evm.model.supercontig_160.33 & AP2-like ethylene-responsive transcription factor ANT & 5.37 & -6.91 & $6.53 \mathrm{E}-04$ \\
\hline evm.model.supercontig_20.162 & Expansin-like A2 & 6.35 & -7.60 & $1.86 \mathrm{E}-04$ \\
\hline evm.model.supercontig_84.92 & Histone H3 & 6.36 & -7.73 & 4.59E-04 \\
\hline evm.model.supercontig_19.182 & cytochrome P450 90A1 (CYP90A1, CPD) & 6.70 & -8.65 & $3.44 \mathrm{E}-05$ \\
\hline evm.model.supercontig_97.108 & COMITIN & 4.66 & -16.92 & 7.80E-04 \\
\hline evm.model.supercontig_21.170 & WUSCHEL-related homeobox 4 & 4.45 & -21.19 & $3.80 \mathrm{E}-03$ \\
\hline evm.model.supercontig_49.19 & Abscisic acid 8 -hydroxylase 4 & 3.51 & -21.94 & $5.76 \mathrm{E}-03$ \\
\hline evm.model.supercontig_25.116 & AP2-like ethylene-responsive transcription factor AIL1 & 2.96 & -60.27 & 7.64E-04 \\
\hline
\end{tabular}

${ }^{a}$ Differentially expressed genes parameters: Fold-Change $(\mathrm{FC})>2$ or $<-2$ and a False Discovery Rate $(\mathrm{FDR})<0.05$

gene only amplified in hermaphrodite 'SunUp' and male 'AU9' flowers, which makes its expression specific for plants with male flower organs, and therefore suggests its participation in male flower organ development in papaya. The evaluation of the expression of CpMS1 by RT-qPCR showed that it was up-regulated in male flowers in comparison with hermaphrodite flowers (Fig. 5a), which might be explained by a different number of flower buds needed for RNA extraction from hermaphrodite than from male plants, due to the considerable difference in size between hermaphrodite flower buds (larger) and male flower buds (smaller) or even due to differences in the developmental stages of the flower buds that composed each sample. No amplification of the CpMS1 gene was detected in any of the female flower samples, supporting the RNA-Seq results and CPMS1 participation on male flower organ development.

Regarding CPMS1 expression on papaya male flower buds of different size, the gene was significantly upregulated in flower buds of 3 to $8 \mathrm{~mm}$ but was not significantly up-regulated in smaller flower buds ( 1 or $2 \mathrm{~mm}$ ), mature flower buds (from 9 to $35 \mathrm{~mm}$ ) or flower organs from open male flowers (petals, sepals or anthers) (Fig. 5b). A detailed comparison between male and hermaphrodite flower buds was not possible due to a lack of flower bud material representing all these different developmental stages $(1$ to $35 \mathrm{~mm}$ ) from hermaphrodite plants. Regardless of the lack of hermaphrodite flower buds for this analysis, 
the expression of CpMS1 was not considered to be significantly different between male and hermaphrodite flower buds according to the previous transcriptome analysis (Tables 1 and 2).

\section{CpMS1: homology analysis and genome location}

The sequence of the gene identified as PHD finger protein MALE STERILITY 1 (CpMS1) in papaya was analyzed and compared to the MALE STERILITY 1 gene found in other species and since its expression was specific for papaya flowers with male organs, its location in the papaya genome was also explored. CpMS1 contained a unique PHD zinc finger motif (Cys4-His-Cys3), located between the amino acid positions 605 and 653 . This protein was highly homologous to other MS1 proteins cloned in other angiosperms plants: Arabidopsis thaliana (AtMS1) (53.18\% identity), Oryza sativa (OsMS1) (45.17\% identity), Hordeum vulgare (HvMS1) (43.80\% identity) and Capsicum annum (CaMS1) (29.33\% identity) (Fig. 6) and which functions have already been well characterized. This gene was located on an autosome (papaya chromosome 02) and no other hit was found for this gene on the papaya genome using cDNA and genomic data. Nevertheless, a single homolog protein was identified in papaya: PHD Finger MALE MEIOCYTE DEATH 1 ('evm.model.supercontig_87.13') or CpMMD1 (Fig. 6), which was also differentially expressed between male and female flower buds of a size $1-6 \mathrm{~mm}$ and hermaphrodite and female flower buds of a size 7-12 $\mathrm{mm}$ (Tables 1 and 2) according to the previous transcriptome analysis. However, CPMMD1 did not group with the rest of the MS1 proteins, which indicates that it might have a different function than the one from CpMS1 (Fig. 6). Unfortunately, the CpMS1 gene was not classified as a candidate for sex determination, because it amplified using the genomic DNA from the three different sex-types which means that this gene is not located on the Y chromosome (Fig. 7), although its expression was sex-biased (specific to male and hermaphrodite flowers), and its genomic sequence was not different among sex-types.

\section{Co-expression network of anther development pathway genes}

A co-expression correlation network was build using all differentially expressed genes and a sub-network was extracted from this network (Additional file 5: Figure S5) using the CpMS1 gene, the genes identified as orthologs of genes known to regulate the expression of MS1 in Arabidopsis thaliana (Table 4) and their first closest neighbors in the total gene network. This correlation subnetwork had 287 nodes and 4127 edges and included 4 clusters of correlated genes (Additional file 5: Figure S5). The first cluster was the biggest, it included 209 nodes and 3462 edges. This cluster also included the CpMS1 gene, as well as orthologs of the transcription factors: Sporocyteless/Nozzle (SPL/NZZ), DEFECTIVE IN TAPETAL DEVELOPMENT AND FUNCTION 1 and ABORTED MICROSPORES. The second cluster included the orthologs of the transcription factors: PISTILLATA (PI) and APETALA 3 (AP3), with a positive correlation between them. The third cluster included the protein CLAVATA 1 (CLV1) and the fourth cluster included the transcription factor DYSFUNCTIONAL TAPETUM (DYT). By analyzing the over-representation of biological process annotations of all the genes found in this sub-network (Additional file 6: Figure S6), the following categories with the highest overrepresentation were found: cellular component assembly involved in morphogenesis, pollen development, pollen wall assembly, external encapsulating structure organization, pollen exine formation and sporopollenin biosynthetic processes (Additional file 6: Figure S6).

\section{Discussion}

Differentially expressed genes among papaya flower sex types were detected at early and late developmental stages. The number of differentially expressed genes between male and female or hermaphrodite and female flowers were higher than the number of differentially expressed genes between male and hermaphrodite flowers. Male and hermaphrodite plants are genetically alike, and both have similar versions of a Y chromosome; which could explain a similar pattern of gene expression observed in their flowers $[6,8]$. Furthermore, a similar pattern of expression during early developmental stages makes sense, because male and hermaphrodite flower development is very similar until anthers are developed $[9,10]$. Nevertheless, the number of differentially expressed genes practically doubled in the latest developmental stage compared to the early developmental stage between male and hermaphrodite plants, which could potentially explain differences observed among sex types.

\section{Differential expression in the anther development pathway}

The major finding of this study was a Male Sterility 1 gene (CpMS1) highly up-regulated in male and hermaphrodite flower buds compared to female flower buds, with tissue (only flower buds) and developmental specific (expressed in male flower buds of 3 to $8 \mathrm{~mm}$ ) expression. Since the differential expression of this gene has not been reported in papaya flower buds before, we explored its regulation and discussed features of this gene. Papaya PHD finger protein MALE STERILITY 1 (MS1), was homologous to Arabidopsis, paprika, rice, and barley MS1 proteins. This gene belongs to the PHD- 


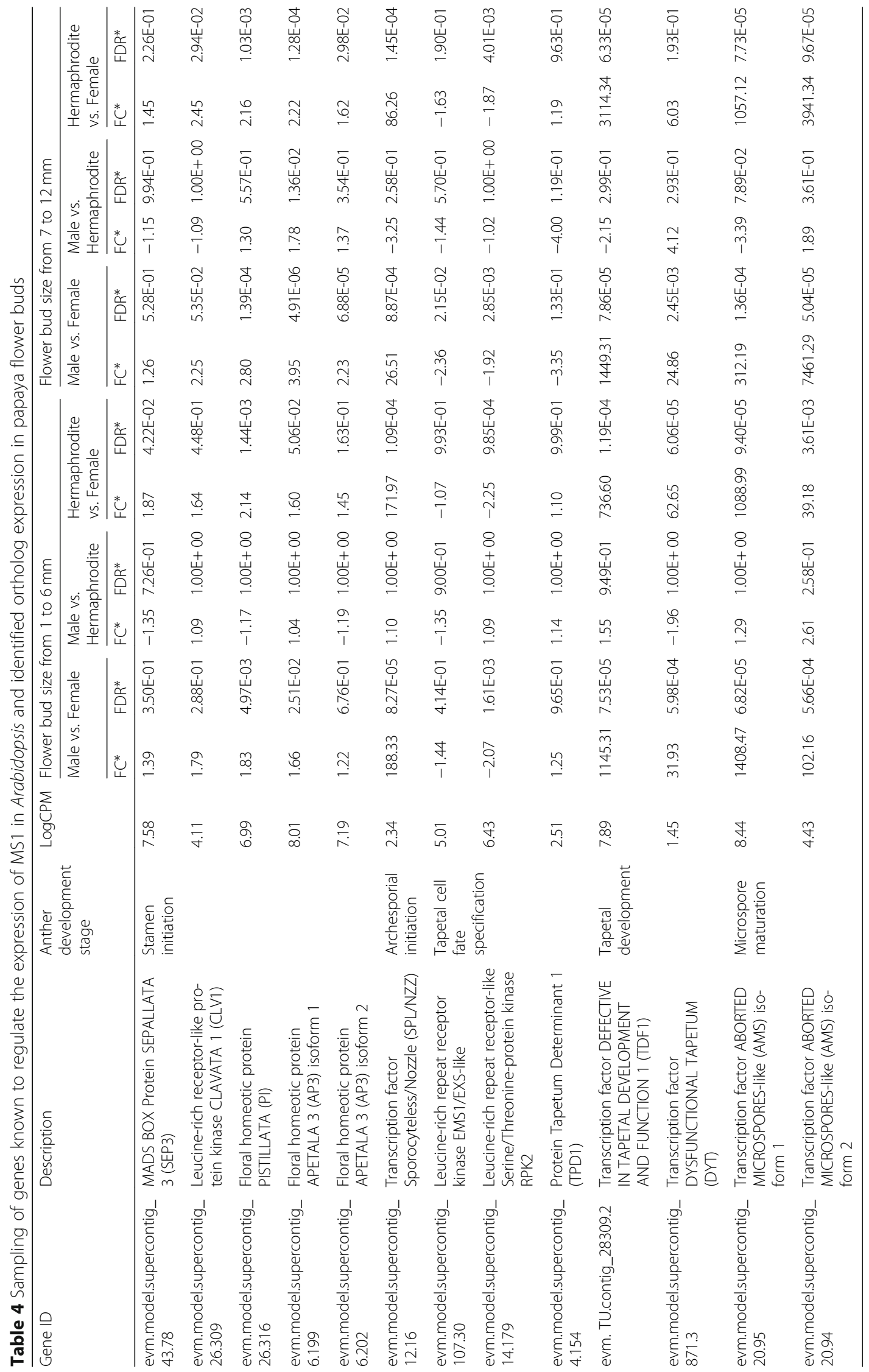




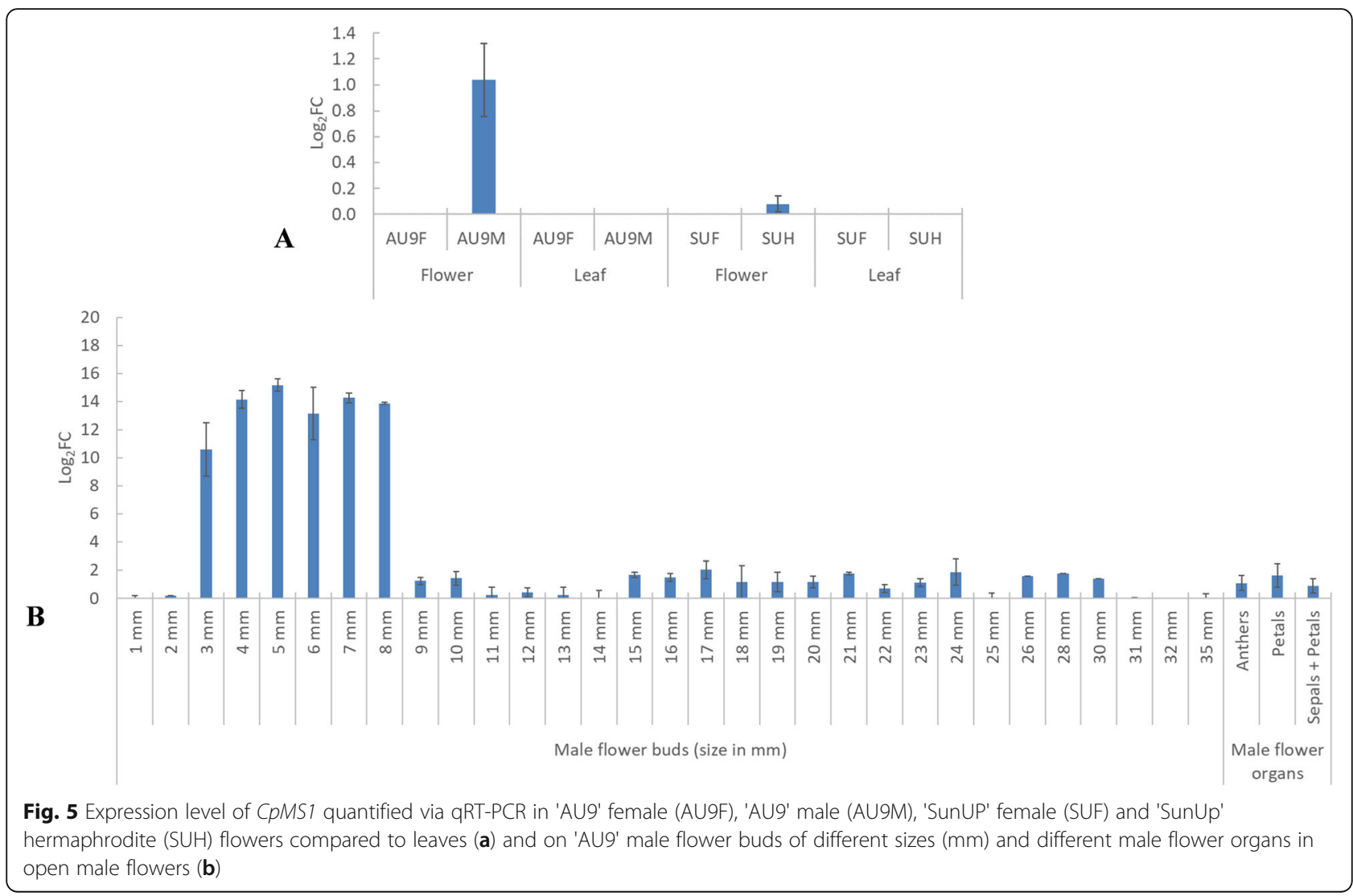

finger family of transcriptions factors. In plants, the PHD (PlantHomeoDomain) transcription factors family has been described as important for several plant development processes, such as pollen maturation, embryo meristem initiation, root development, germination and control of flowering time. It is still unknown what is the specific function of this transcription factor in papaya flowers or its regulation mechanism, but proteins with a PHD motif act as epigenomic effectors, which means that they recognize and bind to histone modifications (e.g. histone methylation), and as a result they activate or repress genes [29]. Little is known about the functions of this protein in papaya, but it is a well-studied gene in other angiosperm species. In Arabidopsis, this gene (AtMS1) has been described as a transcription factor that regulates male gametogenesis, critical for anthers, pollen and tapetum development and it expresses briefly in the tapetal cells during microsporogenesis, just before microspore release [30-34]. In ms1 Arabidopsis mutant plants, the tapetum does not develop correctly, it degenerates abnormally, and the pollen cell wall development is affected; therefore, plants are described as malesterile because their pollen is not viable. This phenotype suggests that MS1 may modify the transcription of tapetal genes participating in pollen cell wall development and tapetal Programmed Cell Death (PCD)
[34]. Genes regulated by MS1 are thought to be involved in the pollen cell wall and coat formation, but this gene also regulates transcription factors involved in pollen production and sporopollenin biosynthesis, as well as certain enzymes (Cysteine proteases) [33]. Over-expression of this gene in Arabidopsis results in plants that show late flowering, flowering stems with an increased number of branches and flowers with distorted organs and reduced fertility [33]. Orthologs of the MS1 gene in Arabidopsis have been described in other plant species: barley (HvMS1) [35], rice (OsMS1) [36] and paprika (CA05g06780) [37], all with a similar function. Therefore, we hypothesize that CpMS1 could have a similar function in papaya due to its homology with the MS1 genes in the other plant species, but more studies are needed to test this hypothesis.

It is important to mention that in other dioecious plant species, like garden asparagus (Asparagus officinalis) and kiwifruit (Actinidia spp.), genes related to early anther development and male sterility have been found as specific candidates for sex determination [38-41]. In asparagus, a transcriptome analysis of male flower buds revealed male-biased expression of several genes involved in pollen microspore and tapetum development [40]. Identifying differentially expressed genes exhibiting 
MS1 MAPKMVIS-- -LGSSRRRRR GEM-LFRFEA FCQPGYPANF AGAGGFRDNV RTLL-GFA-- HLEAGVHGET KCWSFQLEI- ----HRHP-P TVVRLF

HVMS1 MAAKMVIS-D-LGSTR

AtMS1 - MANT TRTDH QQHTPHYRR

CPMS1 -MSNISOT-- -TDLRRRKRK SES-GFRFKT FGEHGCPVEF EGPLPERYNL FALLL-EYG-- NLESNLFGGM QSWSEQLEV- ----HRHP-P FHILLF

CPMMD1 MSIPITET-- ---CRKRRRR PK--IFEFHK FAEPGHPVSF TGP--ERDNV RIFLEECA-- EPEDYYVGGM PIWC-TLIV- ----HENK-S FVLPLIY

OSMS1 EVAASPHRQC HLCRHIGWGR HLICSKRYHF LIPRR-ESAA EADGLCFAIN HGGGGGAEKA SSKGTT---- -------- -TTASSRGHL LHGVVH

HvMS1 EVAASPHRQC HLCRVIGWGR HLICSKRFHF VLPRR-ESSV ETDGLCYGI- ---GGADKAS KGTATS---- - - -

CAMS1 DVSRCRSVYC DQCRVVGWSS NPVCATRFHF IIKADGNSIG GYNRSCSGC- ---GEALHLS ESRCRSCNHV MTTEDVEDWM YHQLEDTSHL LHGVIH

AtMS1 PIEASINLRC NHCQYVGWGN QMTCNKKYHF VIPSK-ETMA A----FLKLE ---GGGYAFP ERESFS---- -------- -HLVELQGHV LHGFFH

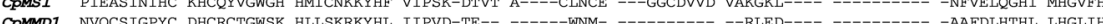

OSMS1 GHLVALHGLE GGSDFVSGHQ TMDLWDRTCS ALHVRTVSLV DTARKGHMEI RLLHGVVAYGE TWFGRWGYRY GRPSYGVALP SYRQSLHVLG SMPLCV

HvMS1 GHLVGLHGFE GGSDFVSGHQ IMDLWDRICS ALHVRRVSLV DTARKGHMVL RLILHGVAYGD TWFGRWGYRY GRPSYGVALQ SYQQSLHALQ SIPLCV

CAMS1 GHLLRINGRE GGSRLLSGSR IINFWDRLCK VLGVRKISGM DVSKKKGLEL RLLHAITKG PWFGEGGF GAGSFALTQD SYRQAVENLS SLPLTI

CPMS1 GHLICINGVE VGSD-LTGHQ IMEFWDRLCT GLKARKVSIN DASQKRGMEL RLIHGVAYGE SWFGHWGYKF GRGTYGITQP VYQKAIEAIQ GMPLCI

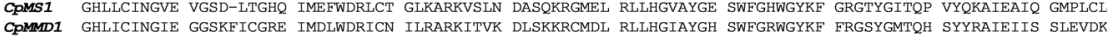

OSMS1 LSC--FSQEL PMVVTKYQAI SGHKLLLSLGD LIRFMLEL-- ---RARLPAT SVTAMDY-RG - -

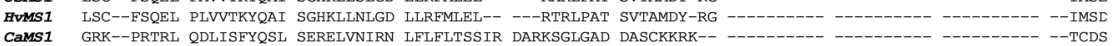

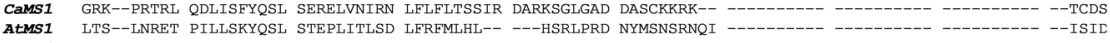

CPMS1 LAN--LNLEI PVIFSRYQTL SDNSLATLGD LFHFMLEL-- ---KSCLPKD QSSIDSYNQG --

CPMMD1 FGNEEFSREI KQIISHYRNK SEAQLITLRD LLRFMLTV-- ---KSCSLSQ NRLAMTITDG PSLMLNSSNR TTLEKHALVR KEQKVIKYRR FSTMVS

OSMS1 RWSAKRVDMA ARAVVDALRR AE-PAARW-- --VTRQEVRD AARAYIGDTG LLDFVLKSL- GNHIVGNYVV RRTMNPVTKV LEYCLEDVSS VLPAV-

$\begin{array}{ll}\text { HvMS1 } & \text { RWSAKRVDMA ARAVVDALRR SEAPAARW-- --VTRQEVRD AARTYIGDTG LLDFVLKSL- GNHIVGNYVV RRAMNPVTKV LEYCLEDVSS VLPASV } \\ \text { CaMS1 } & \text { AWTSSDILRV EEAMFRVLRA VS--GSNW-- --VSWRALRG AVCKA-GSPE LLDYCLKELH GKOAADGMVV NARSVSGSGT MEFKLEPGNP LVNVN- }\end{array}$

CaMS1 AWTSSDILRV EEAMFRVLRA VS--GSNW----VSWRALRG AVCKA-GSPE LLDYCLKELH GKQAADGMVV NARSVSGSGT MEFKLEPGNP LVNVN-

ACMSI RWSARRVEA TRVIVEALKS AE---FRW-- --VSROEVRD AARVYIGDTG LLDFVLKSL- GNOIVGNYLV RRSLNPVTKV LEYCLEKISD VISNO-

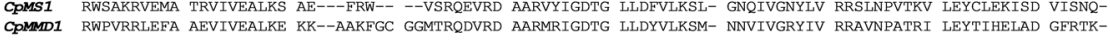

OSMS1 GVPAQGK--- ---MRVRFQL TRAQLMRDLV HLYRHVLKEP SQALTG--GA FGAIPVAVRM VLDIKHFVKD YHEGQAAASS NGGGGFGHPH INLCCT

HvMSI GVPAGHGK-- ---MRVRFHL TRAQLMRDLV HLYRHVLKEP SQALTT--GA FGAIPVAVRM ILDIKHFVKD YHEGMTGTNS GVVGH---VY ISLCCT

CaMS1 RLPCT----- ------PNFP SEEHLRRDLK YLYECMLNPL TMSDHVPLTR REVAVDSATT VLDCKOFMKV YQPERELPIT KS------DA IQVLCE

ATMS1 ITLQNQNSMG KMATNGHNKI TRGQVMKDMF YFYKHILMDY KGVLGPIGGI LNQIGMASRA ILDAKYFIKE YHYIRDTSAK TLHLDRG-EE LGIFCI

CPMS1 VINNSN-C ---VKARYKV TKAQLMKDMF YVFKYILKDH NAAMNT--GL FSAIPVATRV ILDTKYFVKD HYCGEISSKV ELGFE---PK LRLYCT

CPMMD1 EVVQER---- ---IRGPALV PGIDVYSDVT VLYTNLLINY PES------- -ETVETSVQT VLDSKHFVKD WSERDD---- --------KE QTLIVF

OSMS1 ---NGSPELA P----PYETV TLPAHATVGE LKWEAQRVFS EMYLGLRSFA ADSVVGVGAD QEGLPVLGLV DVGSAVVVQG SVGEQINGED HERKEE

HVMS1 ---NGSSELV P----PYETV TVPAHATVGE LKWEVQRLFR DMYLGLRTFT AECVVGIGAG LDASPALGLI GVGSTVVVEG VVGEQQEPAE EGDQRI

CaMS1 -EQSDVHLRN P----PPELI ILPSDATISD LKIETSRTFQ DVYLMFRRFQ ADELVGYGGV KESTQVKLLL GSAEYVTVRG KFLGKNGLS-

CPMS1 GHENTGTTTT KRPLLPYECI TMKSNDTFDD LNT EVGRIFR ETYWGLRNLE VESTVNLNGG KGTDLVFGVV EVGRKIILEG SCKKDGGYVK

CPMS1 GHENTETTT KRPLLPYECI TMKSNDTFDD LNLEVERNFR ETYGLRNIE VESIVNLNGG KGTDLVFGV EVGRKIILEG SCKKDGGVVK -----

OSMS1 AVCEGSGGGE RVVDCACGAV DDDGERMACC DICEAWQHTR CAGIADTEDA PHVFLCSRC- --------- ----DNDVV-- ----SFPSENC

HvMS1 VCEGGGDVGE RVVDCVCGAD DDDGERMACC DICEAWQHTR CAGVADTEDV PHVFLCSRC- --------- ---DNDVA-- ---SFPALNC

CaMS1 RMERG--TER WTVDCYCGAK DDDGERMLAC DVCGVWQHTR CAGISDLDAV PARFICHRCR CVCQTMNTSG NCKDDSVAGA GLGKSLAHAV

A AtMS1 ${ }_{\text {CPMS1 }}$ CYEDDDKKKD KRIECECGAT EEDGERMVCC DICEVWQHTR CVGVQHNEEV PRIFLCQSC- ---------- ---DQHLI-- ---PLSELP-

CPMS1 IYESG--VNN RIVDCPCGGK EDDGERMISC DICEVWQHTR CVRIPNNQEA PHIFLCNRC- --------- ---EREIV-- ---PLSSLP-

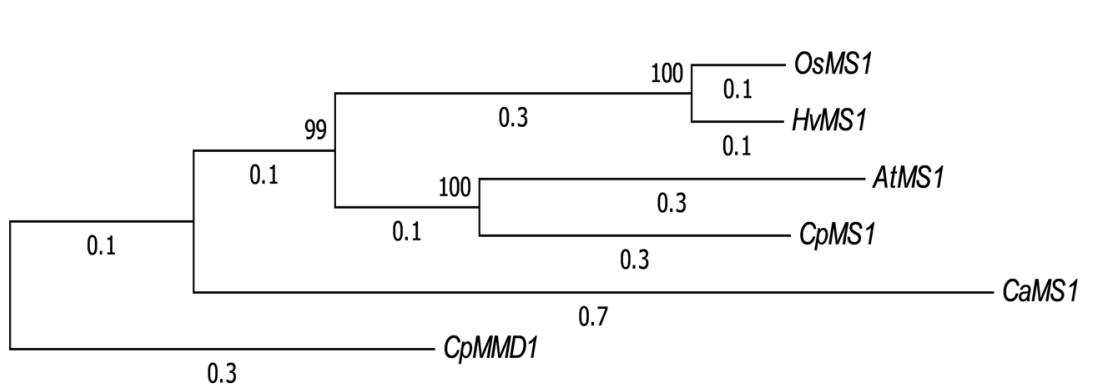

0.3

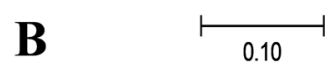

Fig. 6 Alignment of MS1 protein sequences from different plant species (a) and an evolutionary history tree of CpMS1 inferred by the NeighborJoining method using MEGA7 (b)

biased expression in asparagus allowed to identify the earliest points within the anther development pathway that could be influenced by a sex-determination gene. Harkness et al. (2015) showed that in asparagus, microspore maturation genes were up-regulated in male and supermale plants, while down-regulated in females. Later, a MYB-like gene expressed only in asparagus male flower buds, called MALE SPECIFIC EXPRESSION 1 (MSE1), was identified as the sex-determination gene [38]. This gene is homologous to the DEFECTIVE IN TAPETAL DEVELOPMENT AND FUNCTION 1
(TDF1) or MYB35 gene in Arabidopsis, and it is located in the asparagus $\mathrm{Y}$ chromosome [38, 41]. In kiwifruit, a fasciclin-like gene, called Friendly Boy (FrBy) has been identified as a sex- determination gene [39]. This gene is strongly expressed in tapetal cells at early anther developmental stages, which is believed to contribute to tapetum degradation after programmed cell death (PCD) and it is also located on the kiwifruit $\mathrm{Y}$ chromosome [39]. Despite the male and hermaphrodite biased expression pattern observed for the CpMS1 gene, this gene was found to be autosomal, not $\mathrm{Y}$ specific (present in male 


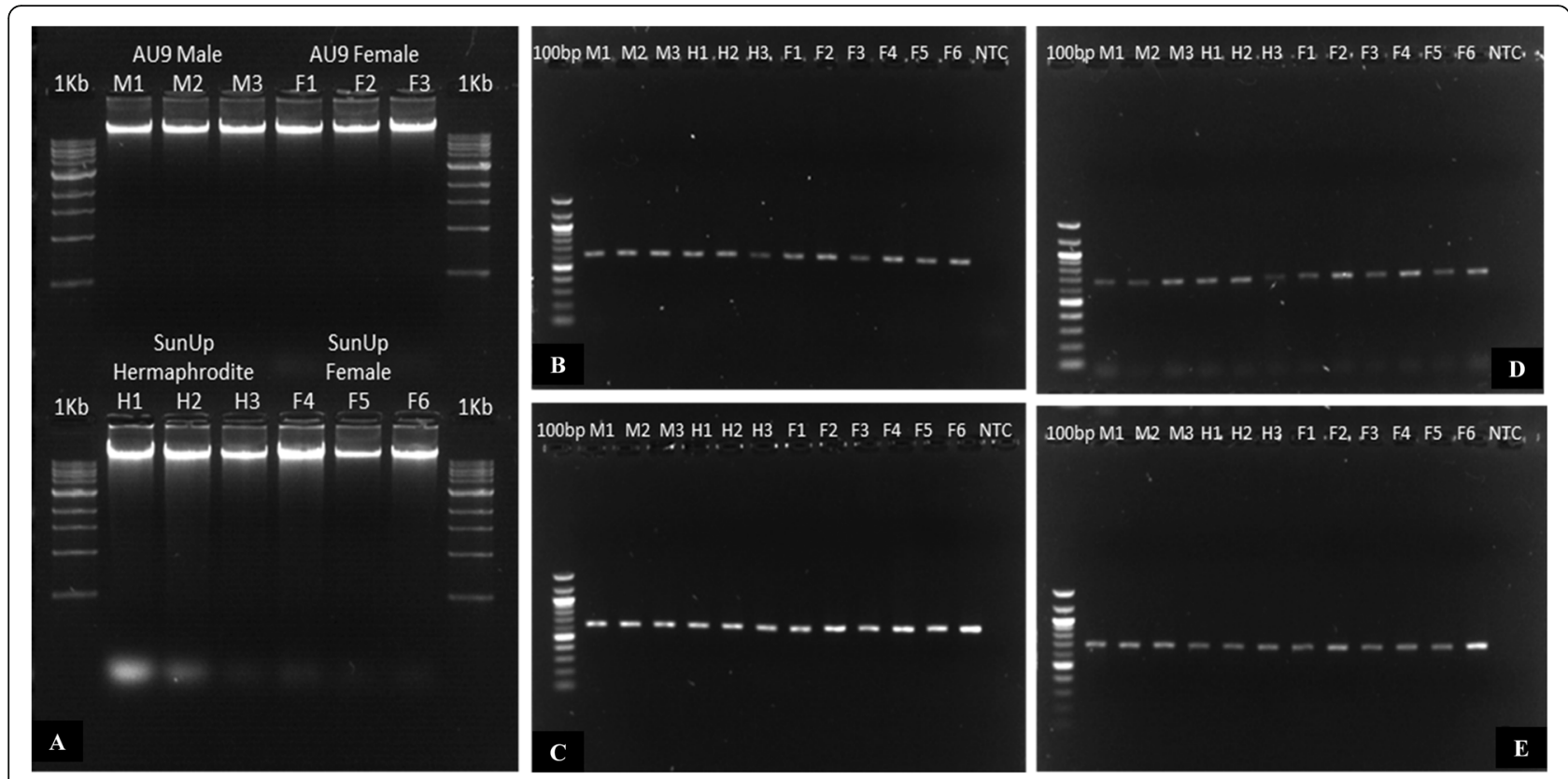

Fig. 7 Amplification of CPMS1 by PCR. a. DNA extracted from female, male and hermaphrodite plants. b. PCR amplification using primers CpMS11F and CpMS1-1R (up) c. PCR amplification using primers CPMS1-2F and Cp MS1-2R (down). d. PCR amplification using primers CpMS1-3F and CPMS1-3R (up). e. PCR amplification using primers CPMS1-4F and CpMS1-4R (down)

or hermaphrodite Y chromosomes), and therefore it cannot be considered as the candidate $\mathrm{Y}$ specific gene for male sex determination in papaya.

Instead, we hypothesize that this gene is playing an important role in male flower organ development, like anther, pollen and tapetum development in early stages of flower development and that it is acting downstream of gender specification. The over-representation of biological processes related to anther and pollen development in the co-expression correlation subnetwork supports our hypothesis. In addition, it has been previously reported that in papaya male flowers, pollen starts to develop in the anthers of flower buds of a size of 0.6 $\mathrm{cm}(6 \mathrm{~mm})$ and tetrads are already found in buds of 0.7 and $0.85 \mathrm{~cm}$ (7 to $8.5 \mathrm{~mm}$ ) [42]. This period overlaps with the expression pattern of the CpMS1 (3 to $8 \mathrm{~mm}$ ). Furthermore, pollen development in papaya has been described to progress at the same pace in all types of pollen-producing flowers, consistently with pollen development in other plants [43, 44]; therefore, upregulation of CPMS1 in small flower buds might be required for tapetum and pollen development in emerging anthers. Nevertheless, more studies are necessary to determine the exact role that CpMS1 is playing in papaya male flower organ development, as well as other genes found as correlated with the MS1 expression in the network.

In Arabidopsis, male flower organ development has been extensively studied and involves a complex network interaction of transcription factors that are expressed in a spatial/temporal manner [45]. MALE STERILITY 1 (MS1) is just one of the last transcription factors involved in this network and it participates in the later stages of tapetum development and pollen cell wall synthesis [33]. Important transcription factors have been reported to act up-stream of MS1 for anther cell specification, like AGAMOUS (AG), SPOROSYTELESS/ NOZZLE (SPL/NZZ), SEPALLATA 3 (SEP3), BARELY ANY MERISTEM 1 (BAM1), BARELY ANY MERISTEM 2 (BAM2) and EXCESS MICROSPOROCYTES1/ EXTRA SPOROGENOUS CELLS (EMS1/ EXS) [45]. Of these transcription factors, only a homologous gene to SPL/NZZ ('evm.model.supercontig_12.16') was identified as differentially expressed between male and female and male and hermaphrodite papaya flower buds (Table 4). The SPL/NZZ gene in Arabidopsis encodes a nuclear protein related to MADS-box transcription factors that are essential to produce most anther cells and to regulate microsporogenesis [46, 47].

Other transcription factors upstream of MS1 participate in tapetal development, like DYSFUNCTIONAL TAPETUM 1 (DYT1), DEFECTIVE IN TAPETAL DEVELOPMENT AND FUNCTION 1 (TDF1), ABORTED MICROSPORES (AMS) and MYB80 [45, 48, 49]. Of these transcription factors, homologous genes to DYT1 ('evm.model.supercontig_871.3'), TDF1 ('evm. TU.contig_28309.2') and two different isoforms of AMS ('evm.model.supercontig_20.94' and 'evm.model.supercontig 20.95') were identified as differentially expressed between male and female and male and hermaphrodite 
papaya flower buds (Table 4). In Arabidopsis, DYT1 encodes a basic helix-loop-helix (bHLH) transcription factor that acts downstream SPL/NZZ and upstream of TDF1, AMS and MS1 [50, 51]. This transcription factor is essential for tapetal gene regulation during tapetal development and it is reported to interact with other bHLH and MYB transcription factors [50, 52]. In Arabidopsis, TDF1 encodes an R2R3 MYB transcription factor required for tapetal development that is regulated directly by DYT1 and act upstream AMS [51]. In Arabidopsis, AMS is a bHLH protein that functions downstream DYT and upstream MS1 and it is essential for pollen development and pollen cell wall synthesis [53, 54]. It is worth to mention here that two MYB transcription factors have been identified in two different inversions on the $\mathrm{Y}$ chromosome [6, 7], but whether these transcription factors participate in any of the steps for anther development in papaya is still unknown.

Overall, the previous results suggest that CpMS1 overexpression observed in male and hermaphrodite flower buds is probably the consequence of a complex regulatory cascade, regulated by a $\mathrm{Y}$ specific gene acting as a stamen promoting factor, as hypothesized by the theory of sex chromosome evolution in plants. More studies are needed to identify the sex-determination gene in papaya on the sex chromosomes that promote male functions.

\section{Other genes found as differentially expressed among different papaya sex-types}

Among the differentially expressed genes annotated as participating in development, reproduction, and embryo development processes between male and hermaphrodite flowers at early stages, we found ABA-8-hydroxylase 1 ('evm.model.supercontig_1525.1'), which was overexpressed in male flowers, and ABA-8-hydroxylase 4, which was overexpressed in hermaphrodite flowers ('evm.model.supercontig_49.19'). Interestingly, the same hydrolases were differentially expressed between normal and teratological male-to-hermaphrodite pistillode, being ABA-8hydroxylase 1 overexpressed in the normal male and ABA-8-hydroxylase 4 overexpressed in teratological male (male-to-hermaphrodite induced plants). Abscisic acid $(\mathrm{ABA})$ is a well-known phytohormone that is involved in the regulation of several plant developmental processes, including seed dormancy and germination, adaptation to environmental stress conditions, mediation of stomatal closure, senescence and flowering time. In Arabidopsis, $\mathrm{ABA}$ induces flowering via drought stress response (DE response) by inducing the up-regulation of GIGANTEA (GI), CONSTANS (CO) and FLOWERING LOCUS T (FT) [55] and inhibits flowering by inducing the upregulation of FLOWERING LOCUS C (FLC) [56, 57]. Interestingly, in male flower buds of a size of 7-12 mm, a GIGANTEA (GI) gene ('evm.model.supercontig_26.81') was up-regulated significantly compared to female flower buds, while in hermaphrodite flower buds of a size of 7$12 \mathrm{~mm}$, a GIGANTEA-like gene ('evm.model.supercontig 26.82 ') was up-regulated significantly compared to female flower buds.

Among other differentially expressed genes between male and hermaphrodite flowers at later stages, we found several transcription factors. A transcription factor annotated as UPBEAT 1 ('evm.model.supercontig $\left.18.81^{\prime}\right)$, was overexpressed in hermaphrodite flowers compared to male flowers at early stages. This transcription factor belongs to the bHLH transcription factor family and has been described to regulate the expression of peroxidases that indirectly determine the concentration of reactive oxygen species (ROS) for the differentiation or proliferation of cells at the root meristems in Arabidopsis [58, 59]. ROS are known to accumulate in response to stress and are important signaling molecules for the regulation of cell division and differentiation in plants [60]. ROS have been also described to participate in different developmental processes in plants, such as programmed cell death (PCD), seed germination, root growth and root hair development, pollen tube growth and leaf development [61]. In olive (Olea europaea L.) hermaphrodite flowers, $\mathrm{ROS}\left(\mathrm{H}_{2} \mathrm{O}_{2}\right.$ and $\left.\mathrm{NO}\right)$ have been reported to accumulate in the reproductive tissues in a developmental dependent manner, with a massive presence on stigmas and anthers, which might be explained by high metabolic activity and cell expansion during the differentiation process [62].

Other transcription factors were overexpressed in hermaphrodite or female flower buds compared to males. Among these transcription factors we found an AP2-like ethylene-responsive transcription factor AIL5 ('evm.model.supercontig_233.1') and a WUSCHELrelated homeobox 4 gene ('evm.model.supercontig 21.170'). AIL5 is an AINTEGUMENTA-LIKE/PLETHORA transcription factor, which is described to play an important role in flower development (especially in floral organ initiation, growth, and patterning), embryogenesis, seedling growth and germination (mediating the repression of gibberellic acid biosynthesis in response to ABA) [63-65]. In Arabidopsis, AIL5 is expressed in developing flowers at specific organs (petals, stamens, and carpels) in a similar pattern to AINTEGUMENTA (ANT), and its overexpression produces larger floral organs $[63,66]$. Overexpression of AIL5 in hermaphrodite and female flower buds compared to male flower buds makes some sense, because hermaphrodite and female flower buds are bigger than male flower buds and they present bigger flower organs [9, 10, 43]. Interestingly, this transcription factor was also differentially expressed between normal and teratological male-to-hermaphrodite 
pistillode, being repressed in normal males and overexpressed in teratological males. WUSCHEL-related homeobox 4 ('evm.model.supercontig_21.170') was found up-regulated between female and hermaphrodite flower buds compared to male flower buds and up-regulated in teratological male (male-to-hermaphrodite) compared to normal male. WUSCHEL-related homeobox (WOX) proteins are transcription factors that belong to the homeobox protein family on the ZIP superfamily and have a variety of functions in plants, including determining cell fate and lateral organ development [67]. In Arabidopsis, 15 WOX genes (including WUSCHEL) have been identified. Some of these WOX genes (including WUSHEL) regulate ovule development, floral organogenesis, floral transition, and participate in gynoecium and embryo development [67, 68]. In Arabidopsis, WUSCHEL also activates the AGAMOUS (AG) gene, a class $\mathrm{C}$ gene required for normal development of carpels in flowers [69-71]. Other WOX genes in Arabidopsis are also capable to alter the expression of the AGAMOUS gene [72].

Here we confirmed the differential expression of important flowering homeotic genes between males or hermaphrodites and females: PISTILLATA ('evm.model. supercontig_26.316') and two AP2-like ethyleneresponsive transcription factor AINTEGUMENTA (ANT) genes ('evm.model.supercontig_129.70' and 'evm.model.supercontig_160.33'), which were also differentially expressed between males and teratological males (maleto-hermaphrodite). It is well known that PISTILLATA (PI) and AINTEGUMENTA (ANT) are required for proper flower organ development in Arabidopsis. PI is required for proper stamen and petal development; while ANT is required for proper flower organ distribution and growth $[66,69,73-76]$. In papaya, the PISTILLATA gene or $C p$ PI has been cloned previously and its expression has been analyzed in male, hermaphrodite and female flower organs. $C p$ PI expression has been reported in petals and stamens of male and hermaphrodite flowers, and only on petals on female flowers [20]. Therefore, this gene was expected to be overexpressed in male and hermaphrodite compared to female flower buds, because female flowers do not present stamens. The down-regulation of $C p$ PI has been reported [16], as well as the up-regulation of two papaya homologous AINTEGUMENTA (ANT) genes, in teratological males (male-to-hermaphrodite) [16], which is consistent with our results. In Arabidopsis, besides its role in floral organ growth, ANT participates in the repression of AGAMOUS (AG) expression in the second floral whorl, promotes petal epidermal cell identity and plays an important role on gynoecium and ovule development [77]. Therefore, overexpression of ANT homologous genes in papaya, in female flowers and teratological male (male-tohermaphrodite) samples compared to males makes sense at early stages of development.

Finally, among differentially expressed genes annotated as participating in development, reproduction, and embryo development processes among male, hermaphrodite and female flowers at early and late stages, we found a VAN3-binding protein. This gene was repressed significantly in male flower buds of $1-$ $6 \mathrm{~mm}$, compared to female flower buds; and in male flower buds of a size 7-12 mm compared to female and hermaphrodite flower buds. In other plants, this protein has been reported to be present in a subpopulation of vesicles from the trans-Golgi-network and to participate in the regulation of the auxin signaling pathway via vesicle transport system [78]. Interestingly, this gene was also differentially expressed in teratological male (male-to-hermaphrodite induced plants) compared to normal male samples. Despite that auxin polar transportation is recognized to play an important role in gynoecium development in Arabidopsis, the specific role of this gene in papaya flower development has not been explored [79, 80].

\section{Conclusions}

Our transcriptomic analysis revealed important differences in the expression of genes that participate in developmental, reproduction and embryo development processes among flower buds from plants with different flower sex type. Even though these genes are not located on the sex chromosomes, their differential expression revealed that more studies on anther development, ABA and ROS signaling pathways are required in papaya, to better understand the roles of these genes in flower development or even in sex determination. It is expected that most of these genes act downstream gender specification in papaya and more studies are needed to determine which sexspecific genes on the sex chromosomes are responsible for sex determination. Furthermore, our results confirmed the expression of a gene: CPMS1 (located on autosomes) in male and hermaphrodite flower buds, which might be required for the normal development of male reproductive organs in papaya. Nevertheless, further studies will be required to elucidate its function and its role in the pathway that regulates male organ development in this species.

\section{Methods}

\section{Plant material}

Flower buds were collected from female and male 'AU9' papaya plants and hermaphrodite 'SunUp' plants grown at the Kunia Research Station of Hawaii Agriculture 
Research Center (HARC) in 2013. Papaya 'AU9' is a breeding plant material originally from Australia and available at HARC; while papaya 'SunUp' is a commercial variety originally from Hawaii available at HARC. The flower buds were used to compare gene expression between sex types and obtain candidate sexdetermination genes by RNA-Seq. These flower buds were first classified according to their phenotype (sex) and then were divided into two groups according to their size (in millimeters). One group contained flower buds with a size between 1 and $6 \mathrm{~mm}$ (early developmental stages, or pre-meiotic stages) and a second group contained flower buds with a size between 7 and $12 \mathrm{~mm}$ (late developmental stages, or post-meiotic stages). Flower buds were ground in liquid nitrogen for further RNA extraction. Two biological replicates were included for each phenotype and for each group. To further corroborate the differential expression of identified highly differentially expressed genes by qPCR, flower buds, and leaf tissue samples were collected again from three different 'SunUp' female plants, three different 'SunUp' hermaphrodite plants, three different 'AU9' female plants and three different 'AU9' male plants grown at the Kunia Research Station of HARC during 2017. These samples were collected and used for the qPCR analysis as described below because original flower bud samples from 2013 were not available. All samples were collected in Hawaii by HARC personnel (no required permissions were necessary to collect the samples), shipped in dry ice $(-80 \mathrm{C})$ to Urbana, Illinois and then ground in liquid nitrogen $(-196 \mathrm{C})$ for further RNA extraction.

\section{Total RNA extraction}

Total RNA was extracted using $100 \mathrm{mg}$ tissue sample and TRIzol ${ }^{\circ}$ Reagent (Ambion USA), following the manufacturer's instructions. After extraction, total RNA was quantified with Nanodrop and its quality was check by electrophoresis (Agarose 1\%, TBE 1X Buffer). RNA samples with good quality and quantity were diluted to 100 $\mathrm{ng} \mathrm{Hl}^{-1}$ and were kept at $-80 \mathrm{C}$ until further use.

\section{RNA-Seq library preparation and sequencing}

RNA-Seq libraries were constructed using 2 to $2.5 \mu \mathrm{g}$ of total RNA and the TruSeq ${ }^{\circ}$ Stranded mRNA LT kit (Illumina USA), following the Low Sample Protocol described by the manufacturer. RNA-Seq libraries were evaluated by electrophoresis (Agarose 1\%, TBE 1X Buffer) and quantified with a fluorometer (Qubit $^{\circ}$ Fluorometer, Invitrogen, USA). RNA-Seq libraries were sequenced using two platforms: HiSeq2000 (single-end, $100 \mathrm{nt})$ for the first biological replicate and HiSeq2500 (pair-end, $100 \mathrm{nt}$ ) for the second biological replicate (Illumina, USA). A summary of the analyzed libraries is presented (Table 5). Besides these libraries, RNA
Sequences from normal male (Accession number: SRX1770718) and teratological male (male-to-hermaphrodite sex reversal induced by low temperatures, Accession number: SRX1770817) from a dioecious variety 'Zhonghuang', were downloaded from the Sequence Read Archive (SRA) on the National Center for Biotechnology Information (NCBI) database [81] and included in the analysis to identify if genes that were differentially expressed in the "pistillode", between males and maleto-hermaphrodite sex reversal plants [16]. Raw sequence data for each library is publicly available on Gene Expression Omnibus (GEO, https://www.ncbi.nlm.nih.gov/ geo/) under the accession number GSE137547 (BioProject: PRJNA565901, SRA: SRP221947).

\section{Differential gene expression analysis}

After RNA sequencing, raw read quality was analyzed using FastQC (Version 0.11.5) [82] and adapters and low-quality reads were removed using Trimmomatic (Version 0.36) [83]. Following trimming, raw reads were aligned to the new papaya genome assembly (Papaya PacBio assembly, 280.5 Mb) using Hisat2 (Version 2.0.5) [84]. After alignment, SAM files were converted to BAM files using samtools (Version 1.3.1) [85] and aligned reads were counted using featureCounts (Version 1.5.2) [86]. Reads aligned to exons were counted and summarized per gene ID. Therefore, an annotation file (gff3 files) was generated using GMAP (Version 2013-11-27). The annotation file was generated using papaya coding sequences from Phytozome v.12 (Cpapaya_113 ASGPBv0.4.cds.fa.gz, Version 12-29-2015) and a new papaya genome assembly (Papaya PacBio assembly, 280.5 $\mathrm{Mb})$. The gff3 files were transformed to gtf files using gffread (Version 0.9.8) to count the number of aligned reads, as described above.

Differential gene expression between samples was analyzed using $\mathrm{R}$ (Version 3.2.3) and Rstudio (Version 1.0.136) with the following packages edgeR (Version 3.12.1), WGCNA (Version 1.51) and limma (Version 3.26.9). The contrast matrix used for the analysis included all pairwise comparisons between all groups. Only the genes with a Logarithmic Fold Change $\left(\log _{2} \mathrm{FC}\right)>1$ or $<-1$ (or a Fold Change $>2$ ) and a False Discovery Rate $(\mathrm{FDR})<0.05$ were consider as truly differentially expressed. A heatmap was built in $\mathrm{R}$ using all identified differentially expressed genes. Gene Ontology (GO) for 2117 selected differentially expressed genes were analyzed with Blast2GO Basic (Version 4.1.9) to reveal GO categories of differentially expressed genes [87-90]. A GO-Slim functional over-representation analysis based on the list of differentially expressed genes in each of the conditions (male vs. female; male vs. hermaphrodite and hermaphrodite vs. female at different sizes 1-6 $\mathrm{mm}$ and 7-12 $\mathrm{mm}$ ) was performed using PANTHER 
Table 5 Sample information and details of each library

\begin{tabular}{|c|c|c|c|c|c|c|}
\hline Sample & Cultivar & Phenotype (sex) & Flower bud size $(\mathrm{mm})$ & Biological replicate & Label & GEO Accession (\#) \\
\hline CP_AU9F_1_R1 & 'AU9' & Female & 1 to 6 & 1 & AU9F.1.R1 & GSM4081661 \\
\hline CP_AU9F_1_R2 & 'AU9' & Female & 1 to 6 & 2 & AU9F.1.R2 & GSM4081662 \\
\hline CP_AU9F_2_R1 & 'AU9' & Female & 7 to 12 & 1 & AU9F.2.R1 & GSM4081663 \\
\hline CP_AU9F_2_R2 & 'AU9' & Female & 7 to 12 & 2 & AU9F.2.R2 & GSM4081664 \\
\hline CP_AU9M_1_R1 & 'AU9' & Male & 1 to 6 & 1 & AU9M.1.R1 & GSM4081665 \\
\hline CP_AU9M_1_R2 & 'AU9' & Male & 1 to 6 & 2 & AU9M.1.R2 & GSM4081666 \\
\hline CP_AU9 M_2_R1 & 'AU9' & Male & 7 to 12 & 1 & AU9M.2.R1 & GSM4081667 \\
\hline CP_AU9M_2_R2 & 'AU9' & Male & 7 to 12 & 2 & AU9M.2.R2 & GSM4081668 \\
\hline CP_SUH_1_R1 & 'SunUp' & Hermaphrodite & 1 to 6 & 1 & SUH.1.R1 & GSM4081669 \\
\hline CP_SUH_1_R2 & 'SunUp' & Hermaphrodite & 1 to 6 & 2 & SUH.1.R2 & GSM4081670 \\
\hline CP_SUH_2_R1 & 'SunUp' & Hermaphrodite & 7 to 12 & 1 & SUH.2.R1 & GSM4081671 \\
\hline CP_SUH_2_R2 & 'SunUp' & Hermaphrodite & 7 to 12 & 2 & SUH.2.R2 & GSM4081672 \\
\hline $\operatorname{SRX} 1770718^{\mathrm{a}}$ & 'Zhonghuang' & Normal male & NA & 1 & ZH.N.M & - \\
\hline $\operatorname{SRX} 1770817^{\mathrm{a}}$ & 'Zhonghuang' & Teratological male & NA & 1 & ZH.T.M & - \\
\hline
\end{tabular}

${ }^{\mathrm{a}}$ Source: data generated by [16] and downloaded from the SRA at NCBI

database [91] and the respective gene ID for the corresponding Arabidopsis homolog, to reveal differential over-represented GO terms between each of condition. To check whether the 2117 differentially expressed genes belonged to a sex chromosome or to an autosome, genes that were differentially expressed were blasted and mapped to the assembled sex chromosomes pseudomolecules $\left(\mathrm{X}, \mathrm{Y}\right.$, and $\left.\mathrm{Y}^{\mathrm{h}}\right)[6,7]$. No match was found and none of the genes could be mapped back to the sex chromosome pseudomolecules.

\section{RT-qPCR expression analysis to validate differential expression of CPMS1}

Total RNA extracted from $100 \mathrm{mg}$ of frozen ground flower buds and leaf tissue samples from wild type 'SunUp' female and hermaphrodite plants; and wild type 'AU9' female and male plants were treated with DNAse I (ThermoScientific) and $2.0 \mu \mathrm{g}$ were converted to cDNA with the High Capacity cDNA Reverse Transcription Kit (Applied Biosystems) in a $20 \mu \mathrm{l}$ reaction, following the steps described by the manufacturer. The relative expression or Fold Change (FC) of the highly differentially expressed gene CPMS1 (identified by RNA-Seq) was evaluated by qPCR using specific primers (Table 5), $10 \mathrm{ng}$ of cDNA and the PowerUp ${ }^{\text {Tw }}$ SYBR $^{\text {Tw }}$ Green Master Mix (Applied Biosystems) in a CFX96 $6^{\mathrm{Tm}}$ Real-Time PCR Detection System (BioRad) with a standard cycling mode (Tm 58C) and including a dissociation curve as a final step. Three biological replicates, three experimental replicates and three non-template controls (NTC) were used. Relative gene expression was normalized against three different internal endogenous genes (Actin 2, EIF1 and TBP1) and the respective variety female sample as reference. The $\Delta \Delta \mathrm{Ct}$ method was used to calculate the relative expression, where Fold Change (FC) for each gene $=2^{\wedge}-(\Delta \Delta \mathrm{Ct})$ and the log Fold Change $=\log _{2}(F C)$. Significant differences in $\log _{2}(\mathrm{FC})$ were analyzed with an ANOVA and a Tukey test $(\alpha=0.05)$. The expression of this gene was also evaluated by RT-qPCR in male flower buds classified in different developmental stages by their respective sizes in millimeters (from 1 to 35 $\mathrm{mm}$ ); and in petals, sepals and anthers from fully developed open male flowers, as described previously. A detailed comparative analysis between male and hermaphrodite flower buds was not possible due to a lack of material representing all the different flower stages ( $1 \mathrm{~mm}$ to $35 \mathrm{~mm}$ ) from hermaphrodite plants.

\section{A highly differentially expressed gene CpMS1: homology analysis and genome location}

Genomic and protein sequences for the highly differentially expressed gene: 'evm.model.supercontig_2.119' (CpMS1) were extracted from Phytozome (v12.1). Three different databases were used to analyze protein motifs present in the protein sequence: PFAM database [92], SMART database [93] and NCBI Conserved Domains Database [94]. BLASTn was used to analyze the position and the number of copies of the gene in the papaya genome. BLASTp was used to find homologous proteins in the papaya genome. The previous and the new papaya genome assembly (Papaya PacBio assembly, $280.5 \mathrm{Mb}$ ) were used to locate and count the number of copies of the gene in the papaya 
Table 6 Primer pairs for RT-qPCR and PCR of CPMS1

\begin{tabular}{llll}
\hline Gene & Primer $\mathrm{F}$ & Primer $\mathrm{R}$ & Tm (C) \\
\hline Actin 2 & TTCCAAGGGTGAGTATGATGAG & ACACAGGACACAAAAGCCAACTA & 58 \\
EIF 1 & AGGCAGGCAAGAGAAGAT & TTCATACCGAGTAGCGATTC & 58 \\
TBP 1 & GGTAGTAGTAGTTAGGTATGTG & GGCAATCTGGTCTCACTT & 58 \\
CpMS1 & ATGGTCAGCTAAGCGAGTG & CGGGTTAAGCTACGACGAA & 58 \\
CpMS1-1 & TTGGAAATAACAAAACGAGAAA & TGGAGAGATTTCTTCAAAATTG \\
CpMS1-2 & GCAATGGCCTCTTGTGT & TCCTGCCTTCCAAAAGATCA & 55 \\
CpMS1-3 & TCAACTCGCTTAGCTGACCA & TGTGAAGGTGGTGTGATGT & 55 \\
CpMS1-4 & AACCCACTTCGACTCCATTG & TTCTCCATTGCAGTGTTCTT & 55 \\
\hline
\end{tabular}

genome. To find out whether this gene was sexspecific or not, primers were designed to amplify the whole gene in segments of $700-800 \mathrm{bp}$ by PCR and DNA from three biological replicates (wild type 'SunUp' female and hermaphrodite plants and wild type 'AU9' female and male plants) were used. A PCR standard $10 \mu \mathrm{l}$ reaction composed by Taq DNA Polymerase with Standard Taq Buffer (NEB), $0.5 \mathrm{ng}$ of DNA and $0.5 \mu \mathrm{M}$ of the four different specific primer pairs for CpMS1 (Table 6) were used in a GeneAmp ${ }^{\circ}$ PCR System 9700 thermal cycler (Applied Biosystems) using the recommended manufacturer thermocycling conditions ( $\mathrm{Tm}$ 55C). All PCR products were sequenced by Sanger Sequencing in the Roy J. Carver Biotechnology Center at the University of Illinois at Urbana-Champaign, assembled using ChromasPro (version 2.1.8), and compared to the CpMS1 genomic reference sequence. Orthologs for this gene in other species (AtMS1, HvMS1, OsMS1, and CaMS1), as well as homologs in papaya, were aligned with MUSCLE [95] and compared to the CPMS1 papaya protein reference sequence using MEGA7 [96].

\section{Co-expression network analysis}

A co-expression correlation network was built in CytoScape [97] using the Expression Correlation App, and the expression matrix containing the normalized expression values for all differentially expressed genes. A subnetwork was extracted from this co-expression correlation network using the genes identified as the orthologs of genes known to regulate the expression of MS1 in Arabidopsis thaliana (Table 4), the CpMS1 gene and all their first closest neighbors in the co-expression network. To determine which biological process was statistically over-represented in this sub-network, a Hypergeometric test with multiple test correction (Benjamini and Hochberg FDR correction) and a significance level of 0.05 was done in CytoScape using the BiNGO App [98].

\section{Supplementary information}

Supplementary information accompanies this paper at https://doi.org/10. 1186/s12870-019-2169-0.

Additional file 1: Figure S1. A cluster of samples based on the normalized LogCPM values (A) and calculated the biological coefficient of variation (B) after RNA-Seq analysis

Additional file 2: Figure S2. Fold enrichment of GO-Slim Cellular component terms identified as over-represented.

Additional file 3: Figure S3. Fold enrichment of GO-Slim Molecular function terms identified as over-represented.

Additional file 4: Figure S4. Fold enrichment of GO-Slim Biological process terms identified as over-represented.

Additional file 5: Figure S5. Co-expression correlation sub-network of genes in the anther developmental pathway.

Additional file 6: Figure S6. Over-represented biological processes in the co-expression correlation sub-network.

Additional file 7: Table S1. Summary of quality control with FastQC, alignment with HISAT2 and read count with featureCounts.

\section{Abbreviations}

ABA: Abscisic Acid; miRNAs: MicroRNAs; RNA: Ribonucleic Acid; RNASeq: Ribonucleic acid sequencing; ROS: Reactive Oxygen Species; RTqPCR: Quantitative Reverse Transcription PCR; SuperSAGE: Improved variant of Serial Analysis of Gene Expression

\section{Acknowledgments}

The authors would like to thank Jennifer Wai, which contributed to the RNA isolation and RNA-Seq library preparation.

\section{Authors' contributions}

All authors have read and approved the manuscript. RM conceived the project; RM, JW designed the experiment; JW and MLW carried out the experiments; DZC analyzed the data; DZC and LY helped with the design and collection of samples for further GPCR analysis. DZC, JN, and RM wrote and corrected the manuscript.

\section{Authors' information}

Dessireé P. Zerpa-Catanho is currently a Ph.D. student in the Plant Biology Department at the University of Illinois Urbana-Champaign and works under the supervision of Ray Ming.

\section{Funding}

This project was supported by the National Science Foundation (NSF) Plant Genome Research Program Award DBI-1546890 to R.M. The NSF grant provided funding for all personnel, all research assistants, and all experimental costs. The experimental costs include: designing the experiment, collecting and transporting samples from Hawaii to Illinois, processing the samples, analyzing and interpreting data and writing the manuscript. 


\section{Availability of data and materials}

The datasets used and/or analyzed during the current study are publicly available on Gene Expression Omnibus (GEO, https://www.ncbi.nlm.nih.gov/ geo/) under the accession number GSE137547 (BioProject: PRJNA565901, SRA: SRP221947)

\section{Ethics approval and consent to participate}

Not applicable.

\section{Consent for publication}

Not applicable.

\section{Competing interests}

The authors declare that they have no competing interests.

\section{Author details}

'Department of Plant Biology, University of Illinois at Urbana-Champaign, Urbana, IL 61801, USA. ${ }^{2}$ Hawaii Agriculture Research Center, Kunia, HI 96759, USA

Received: 2 July 2019 Accepted: 27 November 2019

Published online: 09 December 2019

\section{References}

1. Charlesworth D. Plant sex determination and sex chromosomes. Heredity. 2002;88:94-101.

2. Charlesworth D. Plant sex chromosomes. Annu Rev Plant Biol. 2016;67:397420.

3. Pannell JR. Plant sex determination. Curr Biol. 2017;27:R191-7.

4. Aryal R, Ming R. Sex determination in flowering plants: papaya as a model system. Plant Sci. 2014;217-218:56-62.

5. Ming R, Yu Q, Moore PH. Sex determination in papaya. Semin Cell Dev Biol. 2007;18:401-8

6. VanBuren R, Zeng F, Chen C, Zhang J, Wai CM, Han J, et al. Origin and domestication of papaya $Y^{\mathrm{h}}$ chromosome. Genome Res. 2015;25:524-33.

7. Wang J, Na J-K, Yu Q, Gschwend AR, Han J, Zeng F, et al. Sequencing papaya $X$ and $Y$ h chromosomes reveals molecular basis of incipient sex chromosome evolution. Proc Natl Acad Sci. 2012;109:13710-5.

8. Liao Z, Yu Q, Ming R. Development of male-specific markers and identification of sex reversal mutants in papaya. Euphytica. 2017;213(2):53. https://doi.org/10.1007/s10681-016-1806-z.

9. Ronse De Craene LP, Smets EF. The floral development and anatomy of Carica papaya Caricaceae. Can J Bot. 1999;77:582-98.

10. De R, Craene L, Tréhin C, Morel P, Negrutiu I. Carpeloidy in flower evolution and diversification: a comparative study in Carica papaya and Arabidopsis thaliana. Ann Bot. 2011;107:1453-63.

11. Allan P, Mc Chlery J, Biggs D. Environmental effects on clonal female and male Carica papaya L. plants. Sci Hortic. 1987;32:221-32.

12. Iorns MJ. Observations on change of sex in Carica papaya. Science. 1908;28: 125-6.

13. Kumar A, Jaiswal VS. Sex reversal and fruit formation on male plants of Carica Papaya L. by ethrel and chlorflurenol. Proc Plant Sci. 1984;93:635-41.

14. Kumar A. Feminization of androecious papaya leading to fruit set by ethrel and chlorflurenol. Acta Hortic. 1998;463:251-60. https://doi.org/10.17660/ ActaHortic.1998.463.30

15. Mitra SK, Ghanta PK. Modification of sex expression in papaya Carica papaya L. cv. Ranchi. Acta Hortic. 2000; 515:281-6.

16. Lin $H$, Liao Z, Zhang L, Yu Q. Transcriptome analysis of the male-tohermaphrodite sex reversal induced by low temperature in papaya. Tree Genet Genomes. 2016;12:94. https://doi.org/10.1007/s11295-016-1055-2.

17. Chan YK. Studies on carpellody of stamens in papaya Carica papaya L. MARDI Res Bull. 1984;12(2):157-62.

18. Bogantes-Arias A, Mora-Newcomer E. Influence of genotype and temperature on carpellody of papaya. Agron Mesoam. 2017;28:557-90.

19. Jiménez VM, Mora-Newcomer E, Gutiérrez-Soto MV. Biology of the papaya plant. In: Ming R, Moore PH, editors. Genetics and genomics of papaya. New York, NY: Springer New York; 2014. p. 17-33. https://doi.org/10.1007/978-14614-8087-7 2.

20. Ackerman CM, Yu Q, Kim S, Paull RE, Moore PH, Ming R. B-class MADS-box genes in trioecious papaya: two paleoAP3 paralogs, CpTM6-1 and CpTM6-2, and a PI ortholog CpPI. Planta. 2008;227:741-53.
21. Lee MJ, Yang WJ, Chiu CT, Chen JJ, Chen FC, Chang LS. Isolation and characterization of the papaya MADS-box E-class genes, CpMADS1 and CPMADS3, and a TM6 lineage gene CpMADS2. Genet Mol Res. 2014;13: 5299-312.

22. Liu J, Chatham L, Aryal R, Yu Q, Ming R. Differential methylation and expression of HUA1 ortholog in three sex types of papaya. Plant Sci. 2018;272:99-106.

23. Urasaki N, Tarora K, Shudo A, Ueno H, Tamaki M, Miyagi N, et al. Digital Transcriptome analysis of putative sex-determination genes in papaya Carica papaya. PLoS One. 2012;7:e40904.

24. Yu Q, Hou S, Feltus FA, Jones MR, Murray JE, Veatch O, et al. Low X/Y divergence in four pairs of papaya sex-linked genes. Plant J. 2008;53:124-32.

25. Ueno H, Urasaki N, Natsume S, Yoshida K, Tarora K, Shudo A, et al. Genome sequence comparison reveals a candidate gene involved in malehermaphrodite differentiation in papaya Carica papaya trees. Mol Gen Genomics. 2015:290:661-70.

26. Lee CY, Lin H, Viswanath K, Lin C, Chang B, Chiu P, et al. The development of functional mapping by three sex-related loci on the third whorl of different sex types of Carica papaya L. PLoS One. 2018;13:e0194605.

27. Garg R, Jain M. RNA-Seq for Transcriptome analysis in non-model plants. In: Rose RJ, editor. Legume Genomics. Totowa, NJ: Humana Press; 2013. p. 4358. https://doi.org/10.1007/978-1-62703-613-9_4

28. Wang Z, Gerstein M, Snyder M. RNA-Seq: a revolutionary tool for transcriptomics. Nat Rev Genet. 2009;10:57-63.

29. Mouriz A, López-González L, Jarillo JA, Piñeiro M. PHDs govern plant development. Plant Signal Behav. 2015;10(7):e993253. https://doi.org/10. 4161/15592324.2014.993253.

30. Wilson ZA, Morroll SM, Dawson J, Swarup R, Tighe PJ. The Arabidopsis MALE STERILITY1 (MS1) gene is a transcriptional regulator of male gametogenesis, with homology to the PHD-finger family of transcription factors: MS1 a transcriptional regulator of male gametogenesis. Plant J. 2001:28:27-39.

31. Ito T, Shinozaki K. The MALE STERILITY1 gene of Arabidopsis, encoding a nuclear protein with a PHD-finger motif, is expressed in Tapetal cells and is required for pollen maturation. Plant Cell Physiol. 2002;43(11):1285-92. https://doi.org/10.1093/pcp/pcf154.

32. Ito T, Nagata N, Yoshiba Y, Ohme-Takagi M, Ma H, Shinozaki K. Arabidopsis MALE STERILITY1 encodes a PHD-type transcription factor and regulates pollen and Tapetum development. Plant Cell. 2007;19(11):3549-62. https:// doi.org/10.1105/tpc.107.054536.

33. Yang C, Vizcay-Barrena G, Conner K, Wilson ZA. MALE STERILITY1 is required for Tapetal development and Pollen Wall biosynthesis. Plant Cell. 2007;19:3530-48.

34. Vizcay-Barrena G. Altered tapetal PCD and pollen wall development in the Arabidopsis ms1 mutant. J Exp Bot. 2006;57:2709-17.

35. Fernández Gómez J, Wilson ZA. A barley PHD finger transcription factor that confers male sterility by affecting tapetal development. Plant Biotechnol J. 2014:12:765-77.

36. Qi Y, Liu Q, Zhang L, Mao B, Yan D, Jin Q, et al. Fine mapping and candidate gene analysis of the novel thermo-sensitive genic male sterility tms9-1 gene in rice. Theor Appl Genet. 2014;127:1173-82

37. Jeong H, Tombor B, Albert R, Oltvai ZN, Barabási A-L. The large-scale organization of metabolic networks. Nature. 2000;407:651-4.

38. Murase K, Shigenobu S, Fujii S, Ueda K, Murata T, Sakamoto A, et al. MYB transcription factor gene involved in sex determination in Asparagus officinalis. Genes Cells. 2017:22:115-23.

39. Akagi T, Pilkington SM, Varkonyi-Gasic E, Henry IM, Sugano SS, Sonoda M, et al. Two Y-chromosome-encoded genes determine sex in kiwifruit. Nat Plants. 2019;5:801-9.

40. Harkess A, Mercati F, Shan H-Y, Sunseri F, Falavigna A, Leebens-Mack J. Sexbiased gene expression in dioecious garden asparagus Asparagus officinalis. New Phytol. 2015;207:883-92.

41. Harkess A, Zhou J, Xu C, Bowers JE, Van der Hulst R, Ayyampalayam S, et al. The asparagus genome sheds light on the origin and evolution of a young Y chromosome. Nat Commun. 2017:8:1279.

42. Allan P. Pollen studies in Carica papaya. I. Formation, development, morphology and production of pollen. S.d Afr J Agric Sci. 1963;6:517-30.

43. Saran P, Solanki I, Choudhary R. Papaya: biology, cultivation, production and uses. Volume 1. 1st edition. Florida:CRC Press Taylor \& Francis Group; 2016: 11-52. https://doi.org/10.1201/b18955.

44. Santos LMS, Pereira TNS, de Souza MM, Damasceno Junior PC, da Costa FR, Ribeiro BF, et al. Optical and ultrastructural study of the pollen grain development in hermaphrodite papaya tree Carica papaya L. Braz Arch Biol Technol. 2008;51:539-45. 
45. Verma N. Transcriptional regulation of anther development in Arabidopsis. Gene. 2019;689:202-9.

46. Schiefthaler U, Balasubramanian S, Sieber P, Chevalier D, Wisman E, Schneitz K. Molecular analysis of NOZZLE, a gene involved in pattern formation and early Sporogenesis during sex organ development in Arabidopsis thaliana. Proc Natl Acad Sci U S A. 1999:96:11664-9.

47. Yang W-C, Ye D, Xu J, Sundaresan V. The SPOROCYTELESS gene of Arabidopsis is required for initiation of sporogenesis and encodes a novel nuclear protein. Genes Dev. 1999;13:2108-17.

48. Li D-D, Xue J-S, Zhu J, Yang Z-N. Gene regulatory network for Tapetum development in Arabidopsis thaliana. Front Plant Sci. 2017;8:1559.

49. Zhu J, Lou Y, Xu X, Yang Z-N. A genetic pathway for Tapetum development and function in Arabidopsis. J Integr Plant Biol. 2011;53:892-900.

50. Zhang W. Regulation of Arabidopsis tapetum development and function by DYSFUNCTIONAL TAPETUM1 DYT1 encoding a putative bHLH transcription factor. Development. 2006;133:3085-95.

51. Gu J-N, Zhu J, Yu Y, Teng X-D, Lou Y, Xu X-F, et al. DYT1 directly regulates the expression of TDF1 for tapetum development and pollen wall formation in Arabidopsis. Plant J. 2014;80:1005-13.

52. Zhu E, You C, Wang S, Cui J, Niu B, Wang Y, et al. The DYT1-interacting proteins bHLH010, bHLH089 and bHLH091 are redundantly required for Arabidopsis anther development and transcriptome. Plant J. 2015;83:976-90.

53. Xu J, Ding Z, Vizcay-Barrena G, Shi J, Liang W, Yuan Z, et al. ABORTED MICROSPORES acts as a master regulator of Pollen Wall formation in Arabidopsis. Plant Cell. 2014;26:1544-56.

54. Lou Y, Zhou H-S, Han Y, Zeng Q-Y, Zhu J, Yang Z-N. Positive regulation of AMS by TDF1 and the formation of a TDF1-AMS complex are required for anther development in Arabidopsis thaliana. New Phytol. 2018;217:378-91.

55. Riboni M, Robustelli Test A, Galbiati M, Tonelli C, Conti L. ABA-dependent control of GIGANTEA signalling enables drought escape via up-regulation of FLOWERING LOCUS T in Arabidopsis thaliana. J Exp Bot. 2016;67:6309-22.

56. Wang Y, Li L, Ye T, Lu Y, Chen X, Wu Y. The inhibitory effect of ABA on floral transition is mediated by ABI5 in Arabidopsis. J Exp Bot. 2013;64:675-84.

57. Shu K, Chen Q, Wu Y, Liu R, Zhang H, Wang S, et al. ABSCISIC ACIDINSENSITIVE 4 negatively regulates flowering through directly promoting Arabidopsis FLOWERING LOCUS C transcription. J Exp Bot. 2016;67:195-205.

58. Tsukagoshi H, Busch W, Benfey PN. Transcriptional regulation of ROS controls transition from proliferation to differentiation in the root. Cell. 2010; 143:606-16.

59. Wells DM, Wilson MH, Bennett MJ. Feeling UPBEAT about growth: linking ROS gradients and cell proliferation. Dev Cell. 2010;19:644-6.

60. Schmidt R, Schippers JHM. ROS-mediated redox signaling during cell differentiation in plants. Biochim Biophys Acta BBA - Gen Subj. 1850;2015: 1497-508.

61. Singh R, Singh S, Parihar P, Mishra RK, Tripathi DK, Singh VP, et al. Reactive oxygen species (ROS): beneficial companions of plants' developmenta processes. Front Plant Sci. 2016;7:1299. https://doi.org/10.3389/fpls.2016.01299.

62. Zafra A, Rodríguez-García M, Alché J. Cellular localization of ROS and NO in olive reproductive tissues during flower development. BMC Plant Biol. 2010;10:36.

63. Nole-Wilson S, Tranby TL, Krizek BA. AINTEGUMENTA-like (AIL) genes are expressed in young tissues and may specify meristematic or divisioncompetent states. Plant Mol Biol. 2005;57:613-28.

64. Yano R, Kanno Y, Jikumaru Y, Nakabayashi K, Kamiya Y, Nambara E. CHOTTO1, a putative double APETALA2 repeat transcription factor, is involved in Abscisic acid-mediated repression of gibberellin biosynthesis during seed germination in Arabidopsis. Plant Physiol. 2009;151:641-54.

65. Tsuwamoto R, Yokoi S, Takahata Y. Arabidopsis EMBRYOMAKER encoding an AP2 domain transcription factor plays a key role in developmental change from vegetative to embryonic phase. Plant Mol Biol. 2010;73:481-92.

66. Krizek BA. Ectopic expression of AINTEGUMENTA in Arabidopsis plants results in increased growth of floral organs. Dev Genet. 1999;25:224-36.

67. Lian G, Ding Z, Wang Q, Zhang D, Xu J. Origins and evolution of WUSCHELrelated Homeobox protein family in plant kingdom. Sci World J. 2014;2014:1-12.

68. Deveaux Y, Toffano-Nioche C, Claisse G, Thareau V, Morin H, Laufs P, et al. Genes of the most conserved WOX clade in plants affect root and flower development in Arabidopsis. BMC Evol Biol. 2008;8:291.

69. Bowman JL, Smyth DR, Meyerowitz EM. Genes directing flower development in Arabidopsis. Plant Cell. 1989;1:37-52.

70. Yanofsky MF, Ma H, Bowman JL, Drews GN, Feldmann KA, Meyerowitz EM. The protein encoded by the Arabidopsis homeotic gene agamous resembles transcription factors. Nature. 1990;346:35-9.
71. Mizukami Y, Ma H. Ectopic expression of the floral homeotic gene AGAMOUS in transgenic Arabidopsis plants alters floral organ identity. Cell. 1992;71:119-31.

72. Park SO. The PRETTY FEW SEEDS2 gene encodes an Arabidopsis homeodomain protein that regulates ovule development. Development. 2005;132:841-9.

73. Krizek BA. AINTEGUMENTA-LIKE genes have partly overlapping functions with AINTEGUMENTA but make distinct contributions to Arabidopsis thaliana flower development. J Exp Bot. 2015;66:4537-49.

74. Hill JP, Lord EM. Floral development in Arabidopsis thaliana : a comparison of the wild type and the homeotic pistillata mutant. Can J Bot. 1989;67:2922-36.

75. Krizek BA, Meyerowitz EM. The Arabidopsis homeotic genes APETALA3 and PISTILLATA are sufficient to provide the B class organ identity function. Development. 1996;122:11.

76. Wuest SE, O'Maoileidigh DS, Rae L, Kwasniewska K, Raganelli A, Hanczaryk K, et al. Molecular basis for the specification of floral organs by APETALA3 and PISTILLATA. Proc Natl Acad Sci. 2012;109:13452-7.

77. Krizek BA, Prost V, Macias A. AINTEGUMMENTA promotes petal identity and acts as a negative regulator of AGAMOUS. Plant Cell. 2000;12:1357-66.

78. Koizumi K. VAN3 ARF-GAP-mediated vesicle transport is involved in leaf vascular network formation. Development. 2005;132:1699-711.

79. Nemhauser JL, Feldman LJ, Zambryski PC. Auxin and ETTIN in Arabidopsis gynoecium morphogenesis. Development. 2000;127:3877.

80. Larsson E, Franks RG, Sundberg E. Auxin and the Arabidopsis thaliana gynoecium. J Exp Bot. 2013;64:2619-27.

81. Leinonen R, Sugawara $H$, Shumway $M$, on behalf of the International Nucleotide Sequence Database Collaboration. The Sequence Read Archive. Nucleic Acids Res. 2011;39 Database:D19-21.

82. Andrews S. FastQC A Quality Control tool for High Throughput Sequence Data. 2016. https://www.bioinformatics.babraham.ac.uk/projects/fastqc/. .

83. Bolger AM, Lohse M, Usadel B. Trimmomatic: a flexible trimmer for Illumina sequence data. Bioinformatics. 2014;30:2114-20.

84. Kim D, Langmead B, Salzberg SL. HISAT: a fast spliced aligner with low memory requirements. Nat Methods. 2015;12:357-60.

85. Li H, Handsaker B, Wysoker A, Fennell T, Ruan J, Homer N, et al. The sequence alignment/map format and SAMtools. Bioinformatics. 2009;25:2078-9.

86. Liao Y, Smyth GK, Shi W. featureCounts: an efficient general purpose program for assigning sequence reads to genomic features. Bioinformatics. 2014;30:923-30.

87. Conesa A, Götz S. Blast2GO: a comprehensive suite for functional analysis in plant genomics. Int J Plant Genomics. 2008;2008:1-12.

88. Conesa A, Gotz S, Garcia-Gomez JM, Terol J, Talon M, Robles M. Blast2GO: a universal tool for annotation, visualization and analysis in functional genomics research. Bioinformatics. 2005;21:3674-6.

89. Gotz S, Garcia-Gomez JM, Terol J, Williams TD, Nagaraj SH, Nueda MJ, et al. High-throughput functional annotation and data mining with the Blast2GO suite. Nucleic Acids Res. 2008;36:3420-35.

90. Götz S, Arnold R, Sebastián-León P, Martín-Rodríguez S, Tischler P, Jehl M-A, et al. B2G-FAR, a species-centered GO annotation repository. Bioinformatics. 2011;27:919-24.

91. Mi H, Huang X, Muruganujan A, Tang H, Mills C, Kang D, et al. PANTHER version 11: expanded annotation data from gene ontology and Reactome pathways, and data analysis tool enhancements. Nucleic Acids Res. 2017:45:D183-9.

92. El-Gebali S, Mistry J, Bateman A, Eddy SR, Luciani A, Potter SC, et al. The Pfam protein families database in 2019. Nucleic Acids Res. 2019;47:D427-32.

93. Schultz J. SMART: a web-based tool for the study of genetically mobile domains. Nucleic Acids Res. 2000;28:231-4.

94. Marchler-Bauer A, Derbyshire MK, Gonzales NR, Lu S, Chitsaz F, Geer LY, et al. CDD: NCBI's conserved domain database. Nucleic Acids Res. 2015;43:D222-6.

95. Edgar RC. MUSCLE: multiple sequence alignment with high accuracy and high throughput. Nucleic Acids Res. 2004;32:1792-7.

96. Kumar S, Stecher G, Tamura K. MEGA7: molecular evolutionary genetics analysis version 7.0 for bigger datasets. Mol Biol Evol. 2016;33:1870-4.

97. Shannon P, Markiel A, Ozier O, Baliga NS, Wang JT, Ramage D, et al. Cytoscape: a software environment for integrated models of biomolecular interaction networks. Genome Res. 2003;13:2498-504.

98. Maere S, Heymans K, Kuiper M. BiNGO: a Cytoscape plugin to assess overrepresentation of gene ontology categories in biological networks. Bioinformatics. 2005;21:3448-9.

\section{Publisher's Note}

Springer Nature remains neutral with regard to jurisdictional claims in published maps and institutional affiliations. 